\title{
Interrelationships between Cellular Density, Mosaic Patterning, and Dendritic Coverage of VGluT3 Amacrine Cells
}

\author{
Patrick W. Keeley, ${ }^{1}$ Mikayla C. Lebo, ${ }^{1}$ Jordan D. Vieler, ${ }^{1}$ Jason J. Kim, ${ }^{1}$ Ace J. St. John, ${ }^{1}$ and \\ Benjamin E. Reese ${ }^{1,2}$ \\ ${ }^{1}$ Neuroscience Research Institute, and ${ }^{2}$ Department of Psychological \& Brain Sciences, University of California, Santa Barbara, California 93106- \\ 5060
}

Amacrine cells of the retina are conspicuously variable in their morphologies, their population demographics, and their ensuing functions. Vesicular glutamate transporter 3 (VGluT3) amacrine cells are a recently characterized type of amacrine cell exhibiting local dendritic autonomy. The present analysis has examined three features of this VGluT3 population, including their density, local distribution, and dendritic spread, to discern the extent to which these are interrelated, using male and female mice. We first demonstrate that Bax-mediated cell death transforms the mosaic of VGluT3 cells from a random distribution into a regular mosaic. We subsequently examine the relationship between cell density and mosaic regularity across recombinant inbred strains of mice, finding that, although both traits vary across the strains, they exhibit minimal covariation. Other genetic determinants must therefore contribute independently to final cell number and to mosaic order. Using a conditional KO approach, we further demonstrate that Bax acts via the bipolar cell population, rather than cell-intrinsically, to control VGluT3 cell number. Finally, we consider the relationship between the dendritic arbors of single VGluT3 cells and the distribution of their homotypic neighbors. Dendritic field area was found to be independent of Voronoi domain area, while dendritic coverage of single cells was not conserved, simply increasing with the size of the dendritic field. Bax-KO retinas exhibited a threefold increase in dendritic coverage. Each cell, however, contributed less dendrites at each depth within the plexus, intermingling their processes with those of neighboring cells to approximate a constant volumetric density, yielding a uniformity in process coverage across the population.

Key words: coverage factor; quantitative trait locus; recombinant inbred strain; regularity index; retinal mosaic; Voronoi domain

\section{Significance Statement}

Different types of retinal neuron spread their processes across the surface of the retina to achieve a degree of dendritic coverage that is characteristic of each type. Many of these types achieve a constant coverage by varying their dendritic field area inversely with the local density of like-type neighbors. Here we report a population of retinal amacrine cells that do not develop dendritic arbors in relation to the spatial positioning of such homotypic neighbors; rather, this cell type modulates the extent of its dendritic branching when faced with a variable number of overlapping dendritic fields to approximate a uniformity in dendritic density across the retina.

Received Apr. 28, 2020; revised Nov. 2, 2020; accepted Nov. 5, 2020.

Author contributions: P.W.K. and B.E.R. designed research; P.W.K., J.D.V., and J.J.K. performed research; P.W.K., M.C.L., J.D.V., J.J.K., and A.J.S.J. analyzed data; P.W.K. and B.E.R. edited the paper; P.W.K. and B.E.R. wrote the paper.

This work was supported by National Institutes of Health Grant EY-019968.

The authors declare no competing financial interests.

Correspondence should be addressed to Benjamin E. Reese at breese@psych.ucsb.edu.

https://doi.org/10.1523/JNEUROSCI.1027-20.2020

Copyright $\odot 2021$ the authors

\section{Introduction}

Several interrelated features of neuronal populations are thought to ensure a uniform participation in the local circuitry of the nervous system, including the size of each population, the manner by which their cells are spaced apart from one another, and how they spread their dendritic arbors. The extent to which these demographic traits are correlated varies, depending on the cell type in question. Relationships between these properties of neuronal populations have been most thoroughly studied within the mouse retina (Reese and Keeley, 2015; Keeley et al., 2017a), a structure that lends itself to such analyses by virtue of its layered 
organization. For instance, some retinal cell types minimize proximity between homotypic cells across the retina, varying their intercellular spacing inversely with cellular density (Reese et al., 2005; Whitney et al., 2008), while others have their positioning constrained only by somal size, being otherwise randomly distributed (Keeley et al., 2017b; Keeley and Reese, 2018). Some cell types regulate dendritic outgrowth in relation to their distance to immediate neighbors (Poché et al., 2008; S. C. Lee et al., 2011), while other types extend their dendrites independent of the local density of like-type cells (Farajian et al., 2004). Where such features covary, they may arise through the action of a common biological process during development. Repellent signaling between homotypic cells, for example, may constrain dendritic growth as well as position somata apart from one another (Huckfeldt et al., 2009). The relationship between cellular density and cellular spacing is another example, whereby the process of naturally occurring (programmed) cell death may play a role in eliminating cells that are positioned particularly close to one another, thereby spacing neighbors apart, contributing to the patterning in the somal array as well as defining the final density of neurons within the population (Raven et al., 2003).

The present study has addressed the interrelatedness of these traits for a population of amacrine cells, the vesicular glutamate transporter 3 (VGluT3) amacrine cells. VGluT3 amacrine cells extend their dendrites within the inner plexiform layer (IPL) to occupy both ON and OFF strata (Haverkamp and Wassle, 2004; Johnson et al., 2004). Their dendritic processing is locally isolated, through the depth of the retina as well as across the surface, ensuring a separation of both $\mathrm{ON}$ versus OFF retinal circuitry and retaining precise spatial signaling across the dendritic field (Chen et al., 2017; Hsiang et al., 2017). Uniquely, VGluT3 amacrine cells release both glutamate and glycine to affect different populations of amacrine and ganglion cells (S. Lee et al., 2016; Tien et al., 2016; Jia et al., 2020). Recent studies in our laboratory have shown that the total number of VGluT3 amacrine cells varies widely between different recombinant inbred (RI) strains of mice, indicating that multiple genes participate in the control of this trait (Keeley et al., 2014a). In the present study, we have asked whether the size of this neuronal population is modulated by naturally occurring cell death during development, and whether this cell death might play a role in the establishment of the patterning that is characteristic of this population of cells in the mature mouse retina, using Bax-KO mice. We then considered whether the variation in the density of VGluT3 amacrine cells across those different RI mouse strains correlated with their patterning. We subsequently used three different Bax conditional $\mathrm{KO}(\mathrm{CKO})$ mice to identify the cellular source of the Bax-mediated effect on VGluT3 cell number and patterning. Finally, we examined whether the dendritic arbors of individual VGluT3 amacrine cells are modulated by local VGluT3 cell density, by considering the relationship between dendritic field morphology and proximity to homotypic neighbors.

\section{Materials and Methods}

Animals. Bax-KO (Bax $x^{\text {tm } 1 S j k} / J$, stock \#002994) mice were backcrossed for over 10 generations with C57BL/6J (B6/J) mice, then subsequently maintained by crossing heterozygous mice to produce WT and KO littermates. Three mice of each genotype were used to determine the number and pattering of VGluT3-positive cells (all females, between 76 and $78 \mathrm{~d}$ of age), and 1 mouse of each genotype was used to prepare retinal sections (both males, $55 \mathrm{~d}$ of age). Additionally, 1 mouse of each genotype was used to assess the density and distribution of ribbon synapses in the IPL (both females, $81 \mathrm{~d}$ of age). VGluT3-cre ( $\mathrm{Tg}(\mathrm{Slc17a} 8$ - icre) $1 e d w / S e a l J$, stock \#018147) and Ai9 Cre-reporter mice (Gt(ROSA)

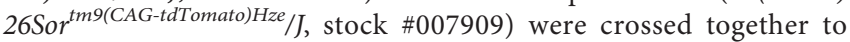
reveal the population of VGluT3 cells (via tdTomato fluorescence) for targeting of single cells for microinjection of dye. Six of these mice were used to analyze the morphology of VGluT3 cells ( 4 females and 2 males, between 42 and $82 \mathrm{~d}$ of age). Additionally, some of these mice were crossed with Bax-KO mice to assess the morphology of VGluT3 cells in $3 \mathrm{KO}$ and $3 \mathrm{WT}$ animals (4 females and 2 males, between 55 and $83 \mathrm{~d}$ of age). Twenty-six RI strains derived from the $\mathrm{B} 6 / \mathrm{J}$ and $\mathrm{A} / \mathrm{J}$ parental strains (the $\mathrm{AXB} / \mathrm{BXA}$ strain set, stock \#001673, \#001674, \#001676, \#001677, \#001678, \#001679, $\# 001681, \# 001683, \# 001684, \# 001685$, \#001687, \#001690, \#001691, \#001692, \#001693, \#001694, \#001696, \#001697, \#001699, \#001700, $\# 001701, \# 001702, \# 001703, \# 001710, \# 001711, \# 001999)$ were used to determine the relationship between VGluT3 cell density and patterning ( 3 or 4 animals per strain, between 42 and $64 \mathrm{~d}$ of age). These RI analyses are derived from a previously published dataset on the total number of VGluT3 amacrine cells in these retinas, in which only female mice had been used, but none of the density nor patterning data have previously been published (Keeley et al., 2014a).

Three different conditional Bax-KO mice (CKO) were generated using Bax-flox mice (Bax ${ }^{\text {tm2Sjk }} B a k 1^{\text {tm1Thsn }} / \mathrm{J}$, stock \#006329; the Bak1$\mathrm{KO}$ allele was removed through selective breeding after receiving the mice from The Jackson Laboratory) and three different Cre-expressing lines targeting different retinal cell types. The first CKO mouse was generated using the aforementioned VGluT3-cre mice to excise Bax from VGluT3 amacrine cells. When crossed to the Ai9 Cre-reporter mice, tdTomato fluorescence is detected in the inner nuclear layer (INL) by the end of the first postnatal week, coincident with when immunostaining reveals the presence of VGluT3 protein. The second CKO mouse

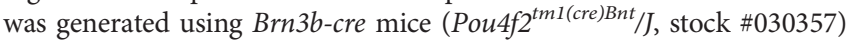
to excise Bax from the population of Brn3b-positive retinal ganglion cells. We have previously analyzed this CKO and confirmed that this population of ganglion cells is almost doubled in size (Keeley et al., 2014b). The third CKO mouse was generated using Vsx2-cre mice ( $T g$ (Vsx2-cre)2690Chow/J, stock \#062600), in which the expression of Cre is restricted to postmitotic bipolar cell precursors (Nickerson et al., 2011), which we confirmed by crossing with Cre-reporter mice. We did find, however, that germline recombination was possible when female mice harboring the transgene were used as breeders; therefore, we used a breeding strategy to maintain the transgene on male breeders only.

Retinas from each CKO line were used to determine the number and patterning of the VGluT3 amacrine cell population, which was compared with that observed in littermate controls (mice that were lacking either the cre allele, the floxed Bax allele, or both; CTRL hereafter). For the VGluT3-cre/Bax-flox mice, 5 CTRL and 6 CKO retinas were examined ( 5 males and 6 females, between 41 and $63 \mathrm{~d}$ old); for the Brn3b-cre/ Bax-flox mice, 3 CTRL and 3 CKO retinas were examined (4 males and 2 females, between 93 and $185 \mathrm{~d}$ old); and for the Vsx2-cre/Bax-flox mice, 4 CTRL and 4 CKO retinas were examined ( 3 males and 5 females, between 57 and $80 \mathrm{~d}$ old). Retinas from one Vsx2-cre/Bax-flox CKO and one littermate CTRL were used to prepare retinal sections for immunofluorescence.

All mice, with the exception of the RI strains, were bred and maintained in the Animal Resource Center at University of California Santa Barbara. All experiments were conducted with approval by the University of California Santa Barbara Institutional Animal Care and Use Committee, and in accord with the National Institutes of Health's Guide for the care and use of laboratory animals.

Tissue preparation. Mice used to quantify cell number and positioning were anesthetized with a lethal dose of sodium pentobarbital ( $\sim 250$ $\mathrm{mg} / \mathrm{kg}$ i.p.; Euthasol; Vibrac). Once deeply anesthetized (as indicated by lack of response to tail pinch), mice were intracardially perfused with $\sim 2 \mathrm{ml}$ of saline followed by $\sim 50 \mathrm{ml}$ of $4 \%$ PFA in $0.1 \mathrm{M} \mathrm{PB}$ (administered over $15 \mathrm{~min}$ via gravity). Eyes were then removed and immersed in fixative for an additional $15 \mathrm{~min}$. Animals used for the single-cell analysis were anesthetized as above, after which eyes were immediately removed and immersed in 4\% PFA. The cornea, iris, and lens were 
quickly dissected from the eyes, and the remaining eyecups were left in the same fixative for an additional $25 \mathrm{~min}$. In both cases, after fixation, whole retinas were removed intact, and four relieving cuts were made radially to allow the retinas to lie flat. Two of these retinal wholemounts were attached to a nitrocellulose membrane and embedded in 5\% agarose in $0.1 \mathrm{M}$ phosphate buffer, then sectioned perpendicular to the retinal surface using a vibrating microtome (PELCO easiSlicer; Ted Pella).

Immunofluorescence. Retinal wholemounts and sections were labeled to detect the presence of the following proteins: vesicular glutamate transporter 3 (VGluT3), synaptotagmin 2 (Syt2), protein kinase A regulatory subunit II $\beta$ (PKARII $\beta$ ), prospero homeobox 1 (Prox1), and C-terminal binding protein 2 (CtBP2). Tissue was blocked in $5 \%$ normal donkey serum (017-000-121; Jackson ImmunoResearch Laboratories) for $3 \mathrm{~h}$, then incubated with primary antibodies for 3 nights, and then finally incubated with secondary antibodies overnight. Between incubations, retinal tissue was rinsed 3 times with PBS, for 10 min per rinse. All solutions were made with a $1 \%$ Triton X-100 in PBS solution, and all steps were performed at $4^{\circ} \mathrm{C}$ on an orbital shaker. The primary antibodies used were as follows: polyclonal goat anti-VGLUT3 (1:500; sc-26031, RRID:AB_2187701; Santa Cruz Biotechnology), monoclonal mouse anti-Syt2 (1:100; ZDB-ATB-081002-25, RRID:AB_10013783; ZIRC), monoclonal mouse anti-PKARII $\beta$ (1:1000; 610625, RRID:AB_397957; BD Biosciences), rabbit polyclonal anti-Prox1 (1:500; PRB-238C, RRID: AB_10064230; Covance), and mouse monoclonal anti-CtBP2 (1:250; 612044, RRID:AB_399431; BD Biosciences). The secondary antibodies used were as follows: polyclonal donkey anti-goat IgG conjugated to AlexaFluor-555 (1:200; A-21432, RRID:AB_141788; Thermo Fisher Scientific), polyclonal donkey anti-mouse IgG conjugated to AlexaFluor647 (1:200; A-31571, RRID:AB_162542; Thermo Fisher Scientific), and polyclonal donkey anti-rabbit IgG conjugated to AlexaFluor-488 (1:200; A-21 206, RRID:AB_2535792; Thermo Fisher Scientific). Hoechst 33342 (1:1000; H3570; Thermo Fisher Scientific) was sometimes added with the secondary antibodies to fluorescently label cell nuclei.

Single-cell labeling. Retinal wholemounts were laid out in a Petri dish with the periphery of each wing held down with a piece of weighing paper (09-898-12A; Thermo Fisher Scientific); this Petri dish was then filled with $0.1 \mathrm{M} \mathrm{PB}$. tdTomato-positive cells were visualized using a fixed-stage fluorescence microscope (Eclipse E600; Nikon) equipped with a $60 \times$ water-immersion objective. A borosilicate glass pipette with a tip diameter of $\sim 0.5 \mu \mathrm{m}$ was filled with Lucifer yellow (L12926; Thermo Fisher Scientific) and then guided into the INL toward fluorescent VGluT3 somata using a micromanipulator (Burleigh PCS-5400; THORLabs). Once the tip of the pipette penetrated the targeted cell, as indicated by passive diffusion of Lucifier Yellow into the soma, a small amount of hyperpolarizing current was passed through the pipette using an amplifier, and the cell was filled with dye until the distal-most dendrites were filled (2-5 min). Multiple tdTomato-fluorescing cells were filled in each retina, which were subsequently immunolabeled for the presence of VGluT3, thereby confirming their VGluT3-positive status, and identifying all of the neighboring VGluT3-positive cells in the field. Filled cells were imaged using a laser scanning confocal microscope (Fluoview1000; Olympus).

Cell counts. Immunostained retinas from the RI strains were analyzed using a fluorescence microscope (BH2; Olympus) attached to a computer running Bioquant software, while retinas from the Bax-WT or Bax-KO animals were imaged with a laser scanning confocal microscope and counted using ImageJ software (imagej.nih.gov/ij). For each retina, eight sample fields were chosen: four in the central retina $(\sim 750 \mu \mathrm{m}$ from the optic nerve head) and four in the peripheral retina $(\sim 750 \mu \mathrm{m}$ from the peripheral edge); each field had an area of $175,000 \mu \mathrm{m}^{2}$ (RI strains), 101,124 $\mu \mathrm{m}^{2}$ (Bax-WT and Bax-KO mice; VGluT3-cre BaxCTRL and Bax-CKO mice; Brn3b-cre Bax-CTRL and Bax-CKO mice), or 44,931 $\mu \mathrm{m}^{2}$ (Vsx2-cre Bax-CTRL and Bax-CKO mice). VGluT3-positive somata were counted in each field from which an average density was determined for each retina. Total estimates of VGluT3 amacrine cell number were derived by multiplying this average density by retinal area. Additionally, the $X, Y$ coordinates of each soma were recorded for every field. Soma size was determined from the Bax-WT and Bax-KO fields by measuring the area of 10 cells in each field, then estimating the diameter of each cell (assuming a circle with identical area) and finding the average diameter for each mouse. Syt2-positive axon terminals and PKARIIB-positive dendritic stalks were quantified to determine Type 2 and Type $3 b$ bipolar cell number, respectively, in the Vsx2-cre Bax-CTRL and Bax-CKO retinas following an identical sampling protocol.

Spatial statistical analysis. To assess the spatial patterning of the VGluT3 amacrine cell population, the Voronoi tessellation was performed for each field using the $X, Y$ coordinates for each soma and a custom program developed in MATLAB (The MathWorks). The Voronoi domain (VD) area for each cell was extracted from this tessellation and used to compute the VD regularity index (VDRI), being the average VD area divided by the SD. Real VDRIs were normalized to the VDRIs calculated from random simulations, in which fields were matched in density and cell placement was constrained only by the average soma size of VGluT3 cells, to derive the VD regularity ratio (VDRR) (Keeley and Reese, 2014). We also measured the nearest neighbor (NN) distance for each cell in every field, and constructed frequency distributions. In all cases, cells near the border of each field whose VD area or $\mathrm{NN}$ distance could not be accurately determined were excluded from the analysis. Spatial statistics were averaged across the eight fields for each retina.

Cell death simulations. One simulation was run for each of the 24 real Bax-KO fields, in which cells were removed from the mosaic sequentially until the density of the field approximated that of Bax-WT fields (specifically, the density of each field was divided by 3.3 to derive the target density of the simulation). Cells were either eliminated by using a uniform random sample (where the probability of any given cell dying is equal) or a weighted random sample (where the probability of any given cell dying is determined by its relative weight). For the latter simulation, relative weights $(W)$ were assigned to each cell $(n)$ based on their average near neighbor distance (aveNND) as follows:

$$
W_{n}=\left(\frac{1}{\operatorname{aveNND}_{\mathrm{n}}}\right)^{k}
$$

Thus, cells that were in closer proximity to neighboring cells had a higher probability of being eliminated than those in sparser regions. Multiple iterations of the simulation, for each field, were thus generated, in which the relative strength of the weighting was adjusted by manipulating the constant $k$ in 0.5 increments, from a value of $1-4$; a value of 3.5 was found to generate simulated fields with spatial characteristics that were comparable with those of Bax-WT fields, presented below in Figure 2.

Quantitative trait locus mapping. VGluT3 cell densities and VDRRs calculated for the RI strains were uploaded in www.GeneNetwork.org as records \#10264 and \#10298, respectively. The interval mapping tool was used to calculate likelihood ratio statistics (LRS) across the genome, and these LRS values were tested for significance using permutation mapping. Quantitative trait loci (QTL), being genomic regions likely contributing to the variation in each trait of interest (density, regularity ratio), were identified as any peak LRS that passed the suggestive threshold established by this permutation mapping.

Morphometrics and plexus analysis. Single dye-filled cells were analyzed for morphologic features using ImageJ. Eccentricity was defined as the distance of each cell from the optic nerve head. Because one group of retinas was slightly larger (on average), and as individual retinas varied slightly in age, eccentricity was normalized by the distance from the optic nerve head to the peripheral retinal margin. Dendritic field area was determined by generating a $z$ axis projection image through the full depth of the arbor in the IPL, then creating a convex polygon that encapsulated the entire dendritic arbor from this image. From these $z$ axis projection images, coverage was calculated as the number of VGluT3 cell bodies underlying the convex polygon (including the filled cell); this was compared with coverages from simulations in which the polygon was rotated around the cell body at three successive $90^{\circ}$ rotations. Additionally, labeled cells were thresholded into binary (black-and-white) images using the levels function in Photoshop (Adobe). Thresholding was performed by eye, so that the threshold image most accurately represented the unaltered image; 
to avoid bias, all 78 images were coded and intermingled so that the thresholder was blind to genotype. The dendritic skeletal volume was calculated by determining the area occupied by the processes of the thresholded dendritic arbor for each optical plane, multiplying by the optical thickness to determine the volume of those processes, and then summing these volumes across the depth of the IPL.

For each field containing a labeled cell, a single optical section was chosen from the middle of the IPL (midway between the INL and ganglion cell layer) to analyze the relationship between the dendritic arbor and the VGluT3-immunopositive plexus. Both the arbor and the plexus were thresholded as before, and the following measurements were calculated from that single plane: dendritic field area (using the convex polygon as describe above), plexus density (the percentage of the sample field occupied by the thresholded VGluT3 signal), and the percentage of the plexus occupied by the dendritic skeleton. Fields in which the VGluT3 staining was overexpressed or underexposed, and therefore may have concealed the true plexus density, were excluded from this analysis, yielding a total of 64 sampled cells.

Experimental design and statistical analyses. All cell counts were conducted blind to genotype, with individual retinas from the RI strains randomly interleaved. All morphometric analyses were conducted blind to genotype, with image stacks for the individual cells also randomly interleaved. All plexus density analyses were conducted blind to genotype, with selected optical sections randomly interleaved. Two-tailed Student's $t$ tests were performed to test for significance between Bax-WT and Bax-KO data, with a threshold for significance of $p<0.05$, indicated by an asterisk in the figures. Pearson correlation coefficients $(r)$ were computed for each scatterplot, and are indicated in the figures. LRS scores associated with the QTL mapping were tested for significance by generating 2000 random permutations of the RI strain data.

\section{Results}

The VGluT3 amacrine cell population undergoes

programmed cell death during development

VGluT3 amacrine cells are situated in the INL, abutting the IPL. They have relatively small somata, being $7.4 \pm 0.05 \mu$ m (mean \pm SEM hereafter) in diameter, and are relatively sparsely distributed, averaging in WT mice 14,368 \pm 138 cells per retina (Fig. $1 a, c)$. In retinas from mice lacking the proapoptotic gene, Bax, soma size was comparable, being $7.3 \pm 0.07 \mu \mathrm{m}$ in diameter, but the total number of VGluT3 cells exhibited a greater than threefold increase, being 51,165 \pm 1349 cells per retina (Fig. 1b,c). While this sample of Bax-KO retinas yielded slightly larger retinal areas (Fig. 1d), most of the increase in total cell number reflected an increase in average VGluT3 cell density, from $906 \pm 11$ to $2984 \pm 58 \mathrm{cells} / \mathrm{mm}^{2}$ (Fig. 1e). This magnitude of change exceeds that for every other retinal cell type documented in the Bax-KO retina (Mosinger-Ogilvie et al., 1998; Péquignot et al., 2003; Whitney et al., 2008; Keeley et al., 2014b), with the exception of the dopaminergic amacrine cells, which exhibit a fourfold to fivefold increase (Whitney et al., 2009; Sankaran et al., 2018). These results demonstrate a role for naturally occurring cell death in defining the size of the VGluT3 amacrine cell population in the mature retina.

\section{The population of VGluT3 amacrine cells is randomly distributed in the $B a x-\mathrm{KO}$ retina}

By identifying the $X, Y$ coordinates of every VGluT3 amacrine cell in each of the eight sampled fields from every Bax-WT and Bax-KO littermate control retina, we computed the Voronoi tessellation of each field (e.g., Fig. $2 a, b$ ), from which we calculated the VDRI (Raven and Reese, 2002). Because somal size necessarily constrains proximity between adjacent cells, whenever such large variation in density exists between two populations one should "normalize" this statistic for each field by calculating the

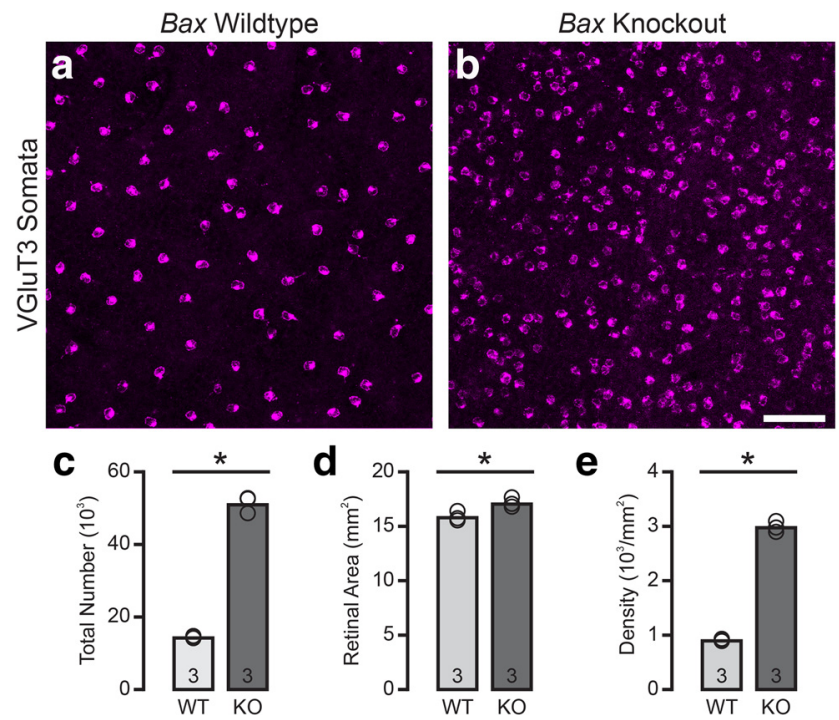

Figure 1. Density of VGluT3 amacrine cells increases in the Bax-KO retina. $\boldsymbol{a}, \boldsymbol{b}$, Example fields of VGluT3-positive somata from a WT littermate mouse $(\boldsymbol{a})$ and a Bax-KO mouse (b), showing the conspicuous increase in VGluT3 amacrine cell density in the Bax-KO retina. c, The total number of VGluT3 amacrine cells undergoes a greater than threefold increase in the Bax-KO retina $(p<0.001)$. $\boldsymbol{d}$, Retinal area was slightly, if significantly, greater in these Bax-KO mice $(p=0.02)$. $\boldsymbol{e}$, The average density of VGluT3 amacrine cells was 3 times greater in Bax-KO mice, confirming that the difference in retinal area contributes only marginally to the difference in total number $(p<0.001) . n=$ the number of retinas sampled in $\mathbf{c}-\boldsymbol{e}$. Scale bar, $50 \mu \mathrm{m}$. $* p<0.05$.

VDRR, being the ratio of the VDRI of the real field to that obtained from a random simulation matched in density and constrained by soma size (Keeley and Reese, 2014). This ratio, in essence, conveys how much more regular a field of cells is relative to a random distribution of space-occupying cells at the same density. We consequently generated random simulations constrained by soma size and density for each individual field, and computed their Voronoi tessellations (Fig. 2c,d), from which we derived their VDRIs, permitting a calculation of the VDRR.

Fields of VGluT3 amacrine cells in Bax-WT retinas appear more orderly in their patterning relative to matched random simulations (Fig. 2a,c), and had higher VDRIs (compare light bars in Fig. $2 e, f)$. Fields from Bax-KO retinas were, by contrast, notably irregular, being hardly different in appearance from their random-constrained simulations (Fig. $2 b, d$ ). If anything, the VDRIs from the $\mathrm{Bax}-\mathrm{KO}$ retinas were slightly lower than were those from the random-constrained simulations (compare dark bars in Fig. 2e,f), likely because, at these greater densities, pairs of cells positioned side-by-side were occasionally closer to one another than average somal diameter (see also Keeley et al., 2017b). As expected, normalizing the VDRI by computing the VDRR confirmed a significant difference between the Bax-WT and Bax-KO retina (Fig. $2 g$ ). We also measured the $\mathrm{NN}$ distances for every cell in each field, to compare their proximity to one another relative to average soma size. In Bax-WT retinas, the average $\mathrm{NN}$ distance was $\sim 22 \mu \mathrm{m}$, whereas in Bax-KO retinas, it was $\sim 11 \mu \mathrm{m}$, being slightly greater than average soma size (Fig. $2 h$ ).

\section{Simulations of proximity-based cell death recreate the patterning in VGluT3 mosaics}

We used the fields of the real Bax-KO retinas to simulate the magnitude of cell loss observed (Fig. 1e), in which the loss of cells was either random or was biased by a proximity-based probability rule (e.g., Fig. $2 i, j$ ). Not surprisingly, the VD analysis of the 

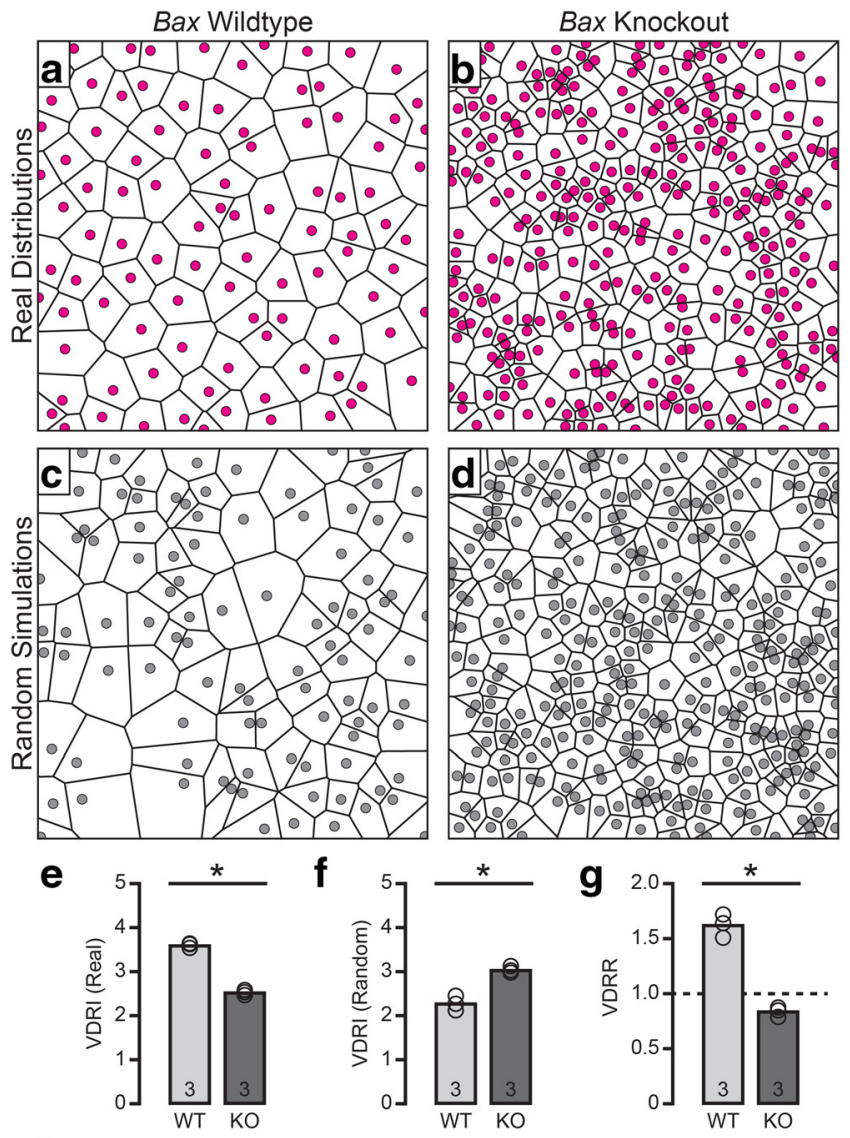

$\mathbf{h}$

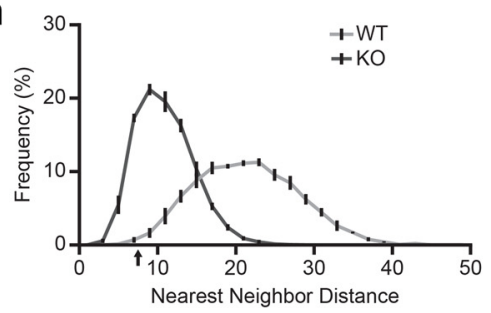

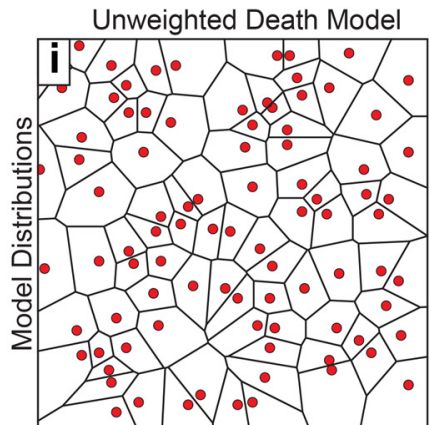

Weighted Death Model
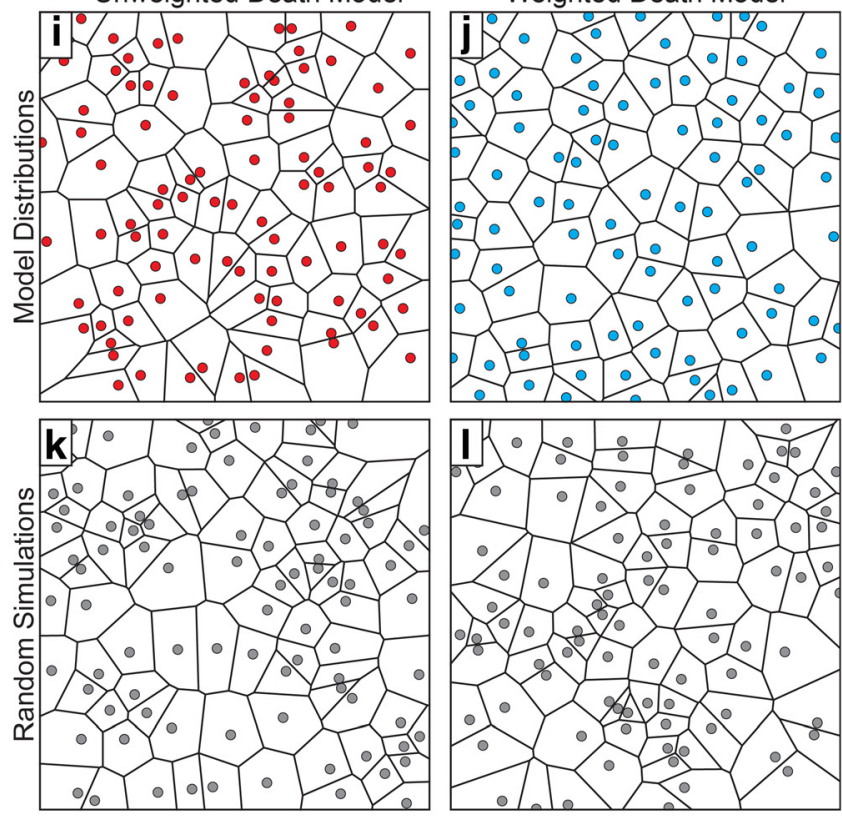

m

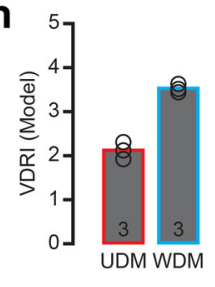

p

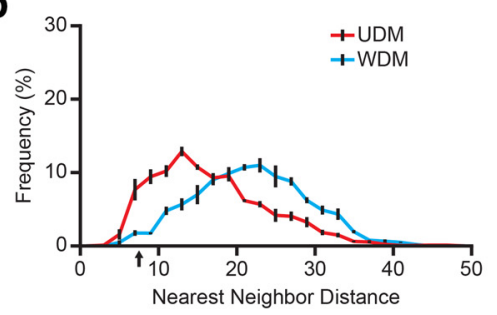

n
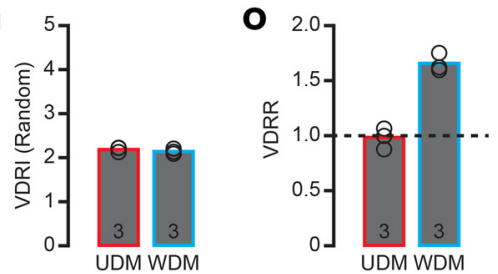

Figure 2. Regularity of the VGluT3 amacrine cell mosaic is abolished in the Bax-KO retina. $\boldsymbol{a}-\boldsymbol{d}$, Voronoi tessellations for the real fields illustrated in Figure $1 a, b(\boldsymbol{a}, \boldsymbol{b})$, and for random simulations matched in density and constrained by soma size (termed "random-constrained simulations" hereafter) (c, $\boldsymbol{d})$. The individual VDs are more uniform in size in the Bax-WT retina $(\boldsymbol{a})$ relative to the random-constrained simulation $(\boldsymbol{c})$. By contrast, there appears to be minimal difference between the Bax-KO retina $(\boldsymbol{b})$ and its random-constrained simulation $(\boldsymbol{d})$. $\boldsymbol{e}$, The VDRI was significantly greater in the WT littermate retinas than in the Bax-KO retinas $(p<0.001)$. $\boldsymbol{f}$, The random-constrained simulations for the WT and KO mosaics revealed the expected effect of cell density alone on the VDRI (Keeley et al., 2020), raising it in the Bax-K0 simulations $(p=0.002)$. $g$, The ratio of these two VDRIs (real/random) for each genotype (being the VDRR) confirmed that the VGluT3 amacrine cells exhibit significantly greater regularity in the WT retina $(p<0.001)$. $\boldsymbol{h}$, Percent frequency distribution of NN distances for the VGluT3 amacrine cell mosaics in Bax-WT versus Bax-KO retinas analyzed in $\boldsymbol{e}$ - $\boldsymbol{g}$. Vertical arrowhead indicates mean soma diameter of $\sim 7.5 \mu \mathrm{m} . \boldsymbol{i}, \boldsymbol{j}$, Voronoi tessellations from the Bax-KO field in $\boldsymbol{b}$ that have been simulated to undergo either random cell death (unweighted death model [UDM]) versus proximity-based cell death (weighted death model [WDM]). $\boldsymbol{k}, \boldsymbol{I}$, Random simulations for respective model fields in $\boldsymbol{i}$ and $\boldsymbol{j}$, permitting the calculation of the VDRR. $\boldsymbol{m}$, VDRIs derived from the two models, showing that a proximity-based death model (WDM) yields a mosaic pattern comparable to the WT retina (compare with $\boldsymbol{e}$ ). $\boldsymbol{n}$, VDRIs are necessarily the same for the random simulations matched in density to the two models. $\boldsymbol{O}$, VDRRs derived from the two models, confirming that the regularity ratio is comparable to that for the WT mosaics (compare with $\boldsymbol{g}$ ). $\boldsymbol{p}$. Percent frequency distribution of NN distances for the mosaics of Bax-KO retinas that had been simulated to undergo random cell death (UDM), or proximity-biased cell death (WDM), analyzed in $\boldsymbol{m}-\boldsymbol{o}$. The distribution for the WDM model approximates the WT distribution in $\boldsymbol{h}$. The distribution for the UDM model differs from that of the Bax-KO distribution in $\boldsymbol{h}$ simply because cell density is 3 times greater in the latter condition (made obvious by comparing $\boldsymbol{a}$ and $\boldsymbol{b}$ with $\boldsymbol{i}$ and $\boldsymbol{j}$ ). $n=$ the number of retinas sampled in $\mathbf{e}-\boldsymbol{g}, \boldsymbol{m}-\boldsymbol{o} . \boldsymbol{h}, \boldsymbol{p}$, Error bars indicate SEM. $* p<0.05$.

Bax-KO mosaic (which we have already shown is comparable to a random distribution; Fig. $2 a-g$ ) that undergoes a random loss of its cells (e.g. Fig. $2 i$ ) yields a VDRI comparable to a random simulated mosaic (Fig. $2 k$ ), in turn generating a VDRR close to 1.0 (Fig. $2 o$; compare with Fig. $2 g$ ). These simulations contained many more side-by-side pairings than in WT fields (Fig. $2 i$; compare with Fig. 2a), despite being matched for density, as evidenced by the distribution of their NN distances (compare the red trace in Fig. $2 p$ with the light gray trace in Fig. $2 h)$. By contrast, depleting such a mosaic by this same magnitude while biasing the elimination based on proximity to other cells yields a mosaic with spatial features not unlike the Bax-WT retinal mosaic (Fig. $2 j$ ), with a VDRR of $\sim 1.7$ (Fig. $2 o$; compare with Fig. $2 g$ ); furthermore, these simulated fields exhibited NN distributions similar to those of Bax-WT fields (compare the blue trace in Fig. $2 p$ with the with light gray trace in Fig. $2 h$ ). Together, these experimental 
and modeling studies suggest that developmental cell death among this population, biased toward eliminating cells in close proximity, bestows on the mature retina the final population of cells distributed in their characteristic array.

\section{RI strains show conspicuous variation} in the density of VGluT3 amacrine cells The total number of VGluT3 amacrine cells varies across the 26 RI strains of the AXB/BXA RI strain set, from a low of $\sim 10,400$ cells per retina to a high of $\sim 14,900$ cells, as previously reported (Keeley et al., 2014a). This variation is largely independent of any variation in retinal area across the strains (Fig. 3a). Rather, most of the variation across the strains is because of variation in average cellular density, from a low of $619 \pm 11$ cells $/ \mathrm{mm}^{2}$ in the BXA25 strain to a high of $931 \pm 25$ cells $/ \mathrm{mm}^{2}$ in the AXB10 strain (Fig. $3 b$ ). As all of these strains are derived from two inbred laboratory strains (B6/J and $\mathrm{A} / \mathrm{J})$, trait differences must arise from variant genes discriminating the two parental genomes. The fact that there is a gradual variation in cell density across the strains makes clear that VGluT3 cell density is a complex trait controlled by multiple genes for which variants discriminate the parental genomes (Reese and Keeley, 2016).

\section{RI strains vary in the regularity of their VGluT3 amacrine cell mosaics}

For each of the eight fields sampled from every retina in each of the 26 RI strains, we generated a random-constrained simulation, and then calculated the VDRIs for the real fields and their corresponding random simulations. From these, we determined the VDRR for each field, and then determined the average VDRR for each strain (Fig. 3c), thereby expressing how different the regularity in each mosaic is relative to a random distribution matched for density and constrained by soma size. For every one of these strains, the ratio is $>1$, yet there is considerable variability across them. The VDRR extends from a low of $1.47 \pm 0.11$ in the BXA12 strain to a high of $1.82 \pm 0.07$ in the BXA11 strain, exhibiting gradual variation across the strain set. The regularity in the patterning of the VGluT3 amacrine cell mosaic, then, must also be polygenic in origin, with genetic variants in many genes contributing to this variation in patterning. Perhaps this variation in patterning, like the variation in density, arises from the same genetic sources that modulate the identical biological process, programmed cell death.

e

f

g

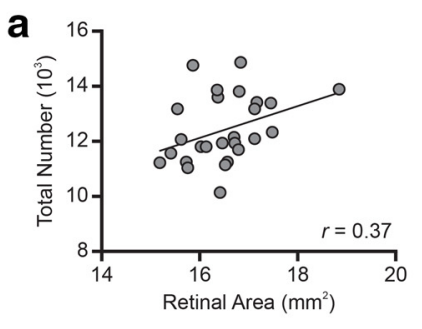

C

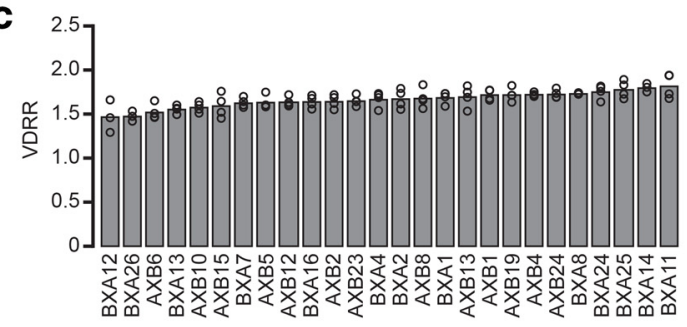

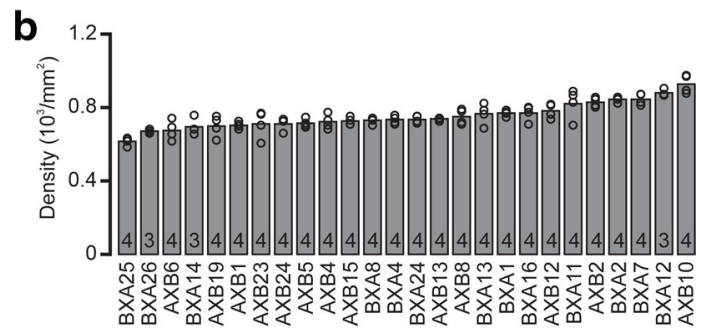

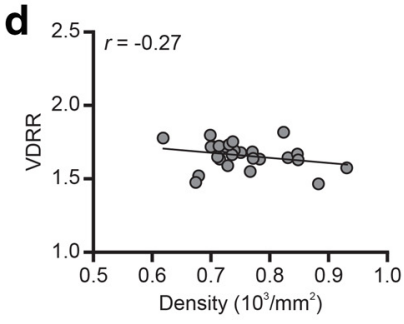

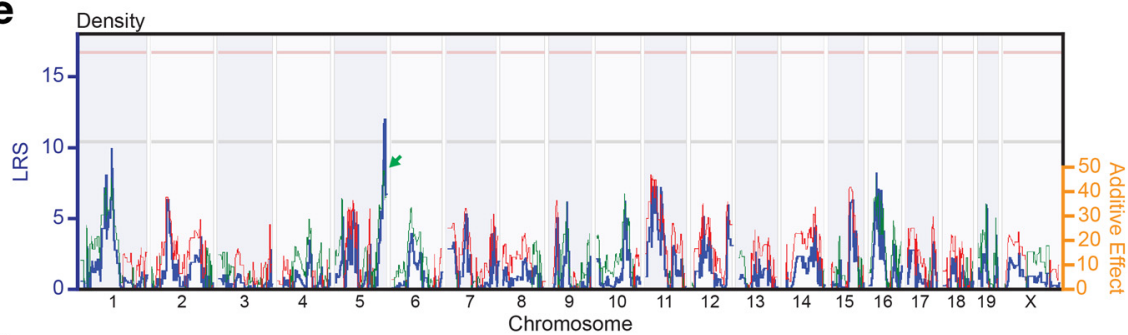
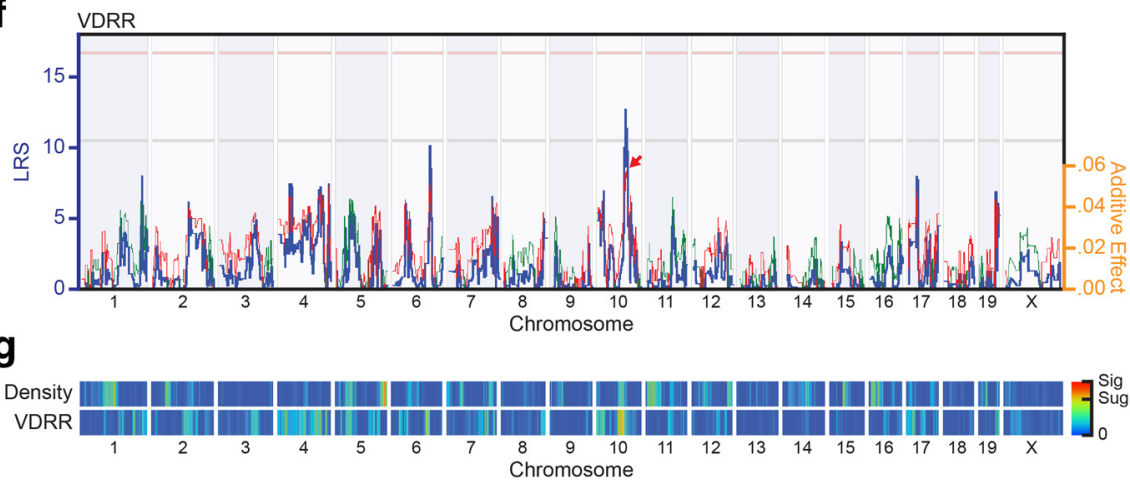

Figure 3. Variation in VGluT3 amacrine cell density is unrelated to the variation in mosaic order across RI strains. $\boldsymbol{a}$, Scatterplot showing the relationship between retinal area and the total number of VGluT3 amacrine cells in the 26 strains of the AXB/BXA RI strain set. Variation in total number is largely independent of the variation in retinal areal size between the strains. $\boldsymbol{b}$, Density of VGluT3 amacrine cells across the 26 RI strains. The variation in total number shown in $\boldsymbol{a}$ is primarily because of these differences in VGluT3 amacrine cell density across the strains. c, The VDRR for VGluT3 amacrine cell mosaics across the 26 RI strains. The regularity of each mosaic, relative to what random simulations of the same density achieve, varies across the strains. $\boldsymbol{d}$, Scatterplot showing the relationship between the average density of VGluT3 amacrine cells within each strain and their regularity ratio (VDRR). Variation in VDRR is largely independent of the variation in cell density across the strains. $n=$ the number of retinas sampled per strain. $\boldsymbol{e}$, Whole genome map for the variation in VGluT3 amacrine cell density. Variation mapped a single suggestive genomic locus on chromosome 5 . Blue trace represents the LRS, indicating the strength of the linkage between genotype and phenotype. Red and green traces represent whether the presence of the $B$ (red) versus $A$ (green) haplotype, respectively, correlates with variation in density across the genome. The right $y$ axis indicates the magnitude of the effect of $B$ versus $A$ alleles across the genome. Green arrow indicates the peak effect size and valence at the chromosome 5 QTL. $\boldsymbol{f}$, Whole genome map for the variation in VGluT3 amacrine cell regularity ratio (VDRR). Variation mapped a single suggestive genomic locus on chromosome 10. Conventions the same as in $\boldsymbol{e}$. Red arrow indicates the peak effect size and valence at the chromosome 5 QTL. $g$, Heat map comparing the variation in LRS score across the genome. Colored regions set lower thresholds to highlight prospective shared genomic control. No shared loci were identified.

Variation in VGluT3 cell density is not predictive of mosaic regularity across the $\mathrm{RI}$ strains

To address the relationship between these two traits across the strains, we first examined the extent to which they covaried. We detected only a slight negative correlation (Fig. $3 d$ ), indicating 
VGluT3-cre / Bax-flox
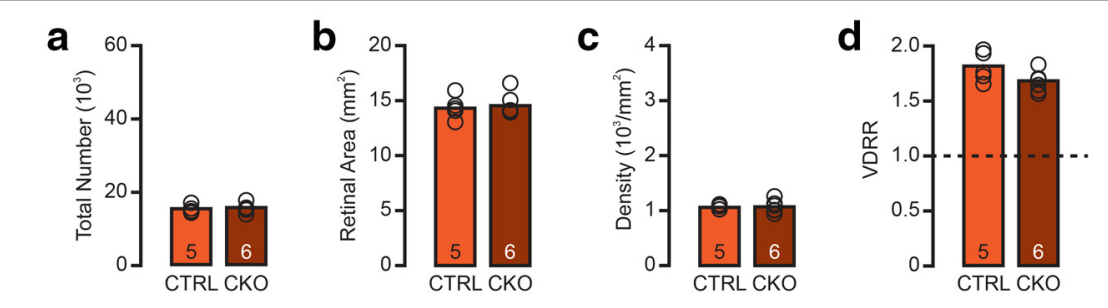

Brn3b-cre / Bax-flox
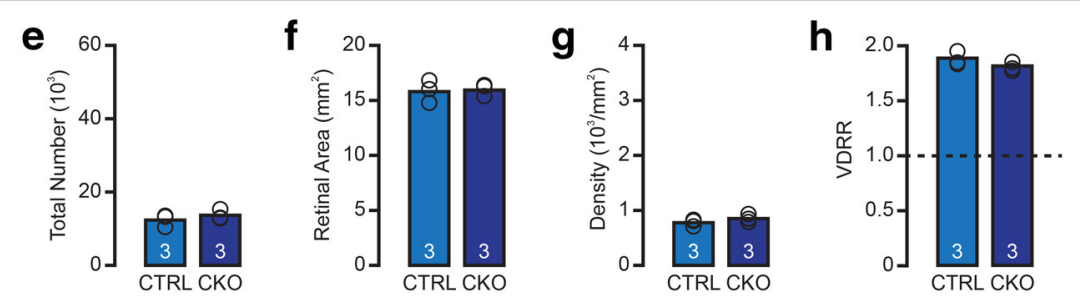

Vsx2-cre / Bax-flox

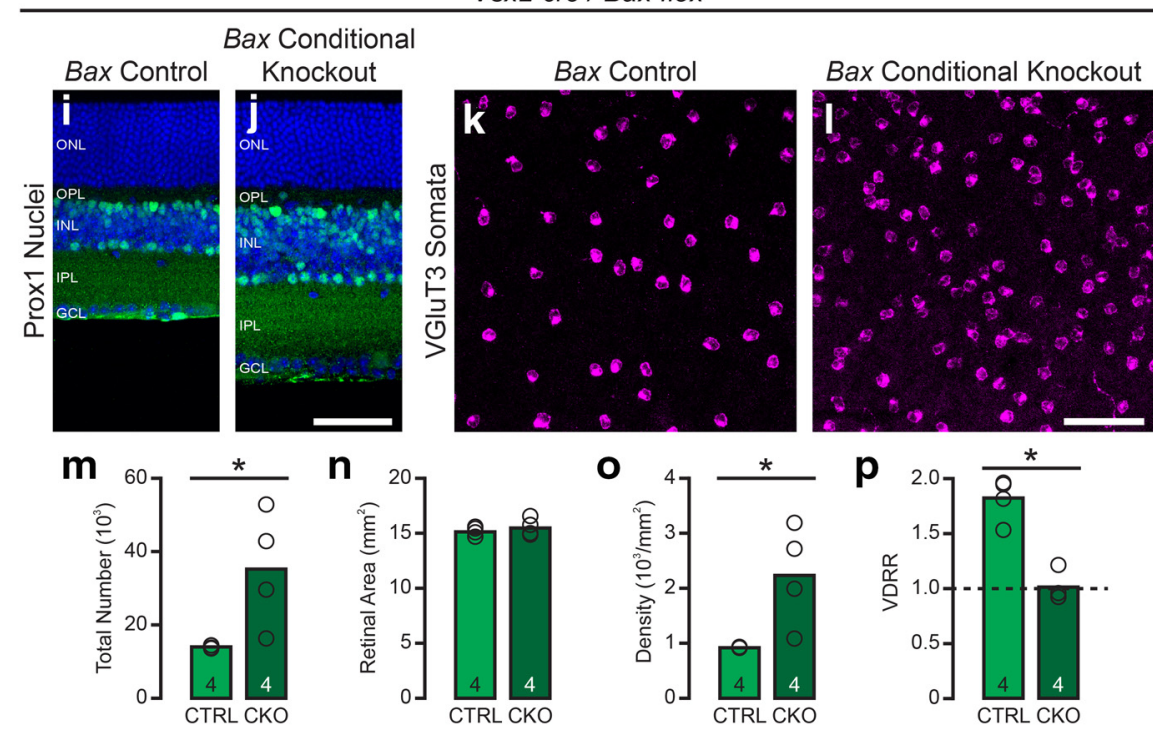

Figure 4. VGluT3 amacrine cell survival is controlled by bipolar cell afferents. $\boldsymbol{a}$ - $\boldsymbol{d}$, The total number $(\boldsymbol{a})(p=0.68)$, retinal area $(\boldsymbol{b})(p=0.71)$, average density $(\boldsymbol{c})(p=0.89)$, and VDRR $(\boldsymbol{d})(p=0.10)$ of VGluT3 cells was unchanged in the VGluT3-cre Bax-CKO retinas. $\boldsymbol{e}-\boldsymbol{h}$, The total number $(\boldsymbol{e})(p=0.35)$, retinal area $(\boldsymbol{f})(p=0.84)$, average density $(\boldsymbol{g})(\boldsymbol{p}=0.24)$, and VDRR (h) $(p=0.18)$ of VGluT3 cells was also unchanged in the Brn3b-cre Bax-CKO retinas. $\boldsymbol{i}, \boldsymbol{j}, 10-\mu \mathrm{m}$-thick cross-sections of retinas from Vsx2-cre Bax-CKO and littermate control retinas immunolabeled for Prox1 (green) and stained with Hoescht (blue). The thickness of the INL has increased, as has the region occupied therein by Prox1-positive bipolar cells in the outer half of the INL. (Prox1 also labels some amacrine cell types, but not the VGluT3 amacrine cells). $\boldsymbol{k}$, $\boldsymbol{I}$, Characteristic fields of VGluT3 cells from Vsx2-cre Bax-CKO ( $\boldsymbol{k})$ and CTRL (I) retinas showing an increase in density and loss of patterning when Bax is eliminated in bipolar cells only. $\boldsymbol{m - 0}$, The total number $(\boldsymbol{m})$ of VGluT3 cells was increased twofold in Vsx2-cre Bax-CKO mice $(p=0.04)$, because of a difference in average density $(\boldsymbol{o})(p=0.03)$, indicated by a largely unchanging retinal area $(\boldsymbol{n})(p=0.45) . \boldsymbol{p}$, Notably, the increased VGluT3 amacrine cell population showed a large reduction in its regularity ratio $(p<0.001)$. 0NL, Outer nuclear layer; $\mathrm{OPL}$, outer plexiform layer; $\mathrm{GCL}$, ganglion cell layer. $n=$ the number of retinas sampled in $\boldsymbol{a}-\boldsymbol{h}$ and $\boldsymbol{m} \boldsymbol{- p}$. Scale bars, $50 \mu \mathrm{m} . * p<0.05$.

that most of the variation in regularity was independent of density. To explore further the possibility that at least some of the variation in these traits might be related, we conducted QTL mapping for each trait. In light of the independent variation in each of these two traits, we expected to map independent primary genomic loci, but nevertheless examined whether any secondary loci might be shared.

The variation in VGluT3 amacrine cell density mapped a single suggestive QTL to the distal end of chromosome 5 from 145.297027 to $146.968082 \mathrm{Mb}(\mathrm{LRS}=12.0)$, where the presence of
$A$ alleles increased cell density by 87 cells per $\mathrm{mm}^{2}$ (Fig. 3e). (This same locus was identified when mapping the variation in total cell number across these same strains; Keeley et al., 2014a). The variation in VGluT3 amacrine cell VDRR, on the other hand, mapped a single suggestive QTL on chromosome 10 from 82.970867 to $84.148124 \mathrm{Mb}(\mathrm{LRS}=12.8)$, where the presence of $B$ alleles increased the regularity ratio by $\sim 0.11$ (Fig. $3 f$ ). Closer examination of the two maps showed no hint of any other loci with an LRS score approaching the suggestive threshold that were shared between the two traits (Fig. $3 g$ ), including the Bax locus itself, on chromosome 7, and composite interval mapping for each trait failed to unveil any other suggestive loci hinting at shared genomic control (not shown). We conclude, therefore, that the genetic variants modulating developmental processes responsible for the variation in local VGluT3 amacrine cell density across this RI strain set must be independent of those variant genes modulating processes that establish the mosaic patterning across these strains.

\section{Programmed cell death of VGluT3 amacrine cells is regulated by their afferents}

Bax-mediated cell death clearly modulates both the cellular density as well as the somal patterning of this population (Figs. $1,2)$, even if it is not the source of the variation of either trait observed across the RI strain set (Fig. 3). The cellular origin of this Bax dependency is, however, uncertain. We consequently examined whether the role of Bax in the first study was acting cell-intrinsically, by assessing mice in which Bax deletion was specific to the VGluT3 amacrine cell population, using VGluT3-cre Bax-CKO mice. No evidence for a cell-intrinsic effect of Bax on VGluT3 cell number was obtained, however, as the density of cells was nearly identical between the CKO and littermate CTRL mice (Fig. $4 a-c$ ). Not surprisingly then, their mosaics exhibited comparable order as well (Fig. $4 d$ ).

There is one potential caveat about this study: Cre activation might occur too late in these retinas to be abrogating any cell-intrinsic effect of Bax in VGluT3 cells. We tested this by examining the developmental onset of tdTomato fluorescence in VGluT3-cre Cre-reporter retinas, finding positive labeling only after P8 (data not shown). This coincided with the onset of VGluT3 expression in the retina, as immunostaining for the protein was detectable only after P7 (data not shown). This analysis was, consequently, inconclusive in determining a cell-intrinsic role for Bax in establishing the number and patterning of the VGluT3 amacrine cells. If, 
however, Bax was not playing such a direct role within the population of VGluT3 cells, the above results in the Bax-KO retina would then have to arise through a dependency on some other cell type undergoing Bax-mediated cell death, presumably either their target neurons or their afferents, which we examined next.

First, we used Brn $3 b$-cre to excise Bax from the population of developing retinal ganglion cells, which we have previously found yields a retina containing a twofold increase in the number of Brn3b-positive retinal ganglion cells (Keeley et al., 2014a; see also Puñal et al., 2019). These Brn3b-cre Bax-CKO retinas contained a population of VGluT3 amacrine cells that was no different in size nor in patterning from their littermate controls containing normal numbers of retinal ganglion cells (Fig. $4 e-h$ ). We then examined the effect of conditionally deleting Bax from bipolar cells, using Vsx2-cre Bax-CKO mice, in which Cre expression is restricted to postmitotic bipolar cell precursors (Nickerson et al., 2011). In these mice, we confirmed an increase in the thickness of the INL containing many more Prox1-positive bipolar cells (Fig. 4i,j), known to be expressed by multiple bipolar cell types (Shekhar et al., 2016), and we quantified an increase in the number of one particular type of retinal bipolar cell, the Type 3b OFF bipolar cell, by 28\% (CTRL: 68,061 \pm 3005 cells, CKO: $87,431 \pm 3318$ cells; $n=4$ retinas per group; $p=0.005$ ), but found no change in another cell, the Type 2 OFF bipolar (CTRL: 57,581 \pm 428 cells, CKO: $57,893 \pm 4385$ cells; $n=4$ retinas per group; $p=0.95)$. In these retinas, we found that the VGluT3 cell population underwent a greater than twofold increase (Fig. $4 k, l)$, although there was large variability in the size of the population across the four CKO retinas analyzed (Fig. $4 m-o$ ). The VDRR was substantially reduced, to nearly the same value in the Bax-KO retina, yielding an average VDRR of $\sim 1.0$ (Fig. $4 p$; compare with Fig. $2 g$ ), indicating that the patterning of the VGluT3 population in these retinas is essentially random. These results demonstrate an afferent dependency of the VGluT3 population by some subset of the different bipolar cell types. This suggested dependency, therefore, would control cell survival among the VGluT3 population, and when cell death among that population is abrogated because of this surfeit of afferents, normal pattern formation in the VGluT3 mosaic fails to materialize.

Given the lack of tight control on the patterning of VGluT3 amacrine cells across the RI strains, as well as their conspicuous loss of patterning in the Bax-KO retina, what then might be the functional consequences for how these cells extend their processes within the IPL? Might these cells simply operate an intrinsic growth strategy, developing dendritic arbors of a particular size independent of their proximity to local neighbors? They would, consequently, exhibit variability in the degree of dendritic overlap because of variation in average cell density, as well exhibit variability in their uniformity of dendritic overlap because of the variation in cell patterning. Alternatively, these cells may regulate the growth of their dendritic arbors to compensate for one or both of these sources of variability, thus maintaining a constant dendritic coverage. To test these possibilities, we analyzed the dendritic morphology of individual VGluT3 amacrine cells with respect to the surrounding population of homotypic cells.

\section{VGluT3 dendritic field areas are independent of VGluT3 amacrine cell density in the WT retina}

The dendritic arbors of individual VGluT3 amacrine cells arise from a single stalk that emerges from the soma, ramifying broadly to contribute processes throughout the IPL. They are too
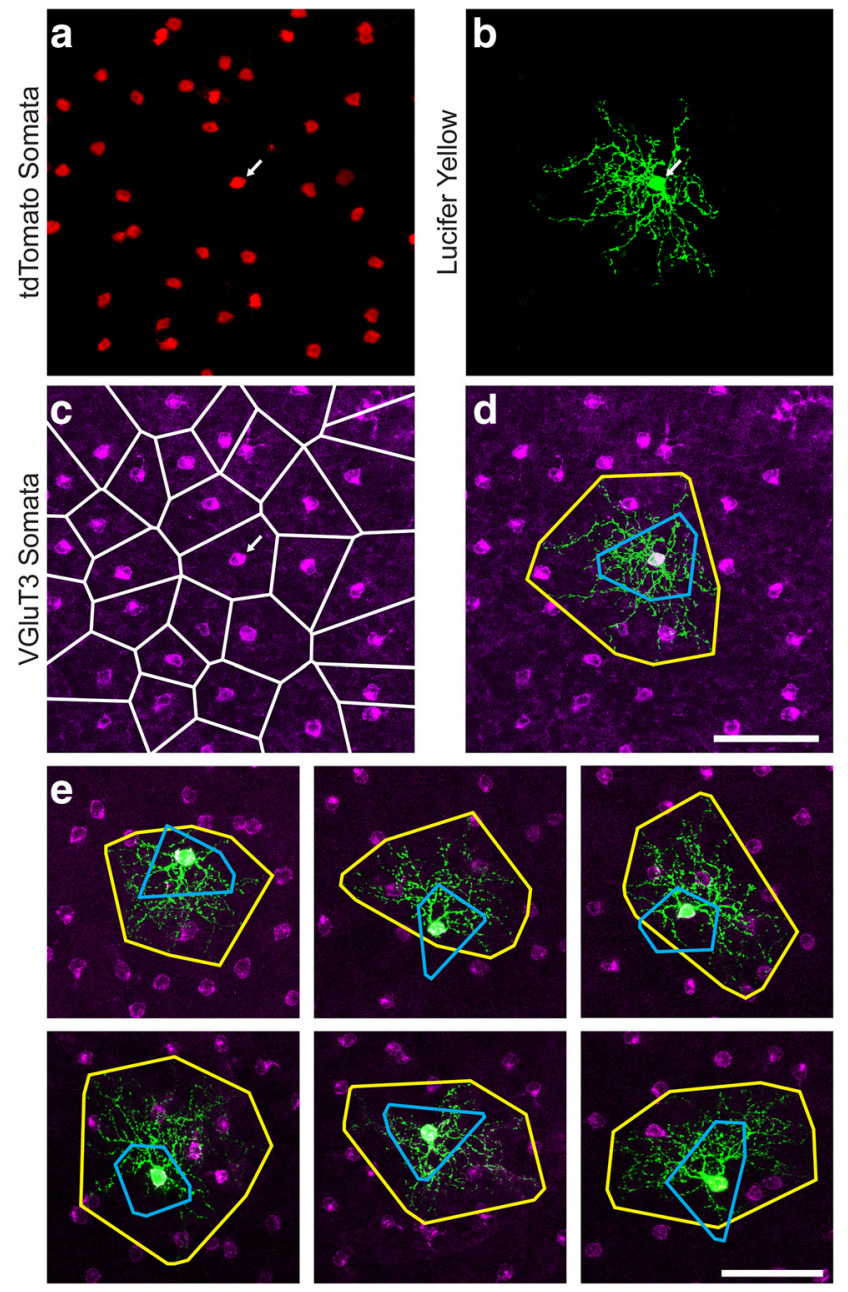

Figure 5. The morphology of single VGluT3 amacrine cell dendritic arbors was compared with their VDs. $\boldsymbol{a}-\boldsymbol{e}$, Single VGluT3 amacrine cells, identified by their tdTomato labeling in VGluT3-cre reporter mice $(\boldsymbol{a})$, were targeted for injection of Lucifer yellow to fill their dendritic arbors $(\boldsymbol{b})$. Retinas were subsequently labeled with antibodies to VGluT3 to confirm the VGluT3-immunopositive status of single injected cells (c). The Voronoi tessellation of the field was determined, and the VD of each injected cell (cyan outline) was compared with its dendritic field area (amber outline) (d). Six additional examples from the total sample of 66 cells are illustrated, showing the variation in both dendritic field orientation and in the size and shape of the VD (e). Multi-color images are composite images to illustrate to relevant features of each signal. Scale bars, $50 \mu \mathrm{m}$.

large, relative to average cell density, to provide a simple tiling (i.e., nonoverlapping but complete coverage) of the retinal surface (Grimes et al., 2011; Kim et al., 2015). Rather, they reach into one another's dendritic territories, a feature common to most other amacrine cell types (Diamond, 2017). Even when dendritic arbors overlap, they may still regulate the areal extent of their dendritic growth in relation to homotypic density, as do horizontal cells (Reese et al., 2005, 2011; Poché et al., 2008). We have, consequently, examined whether the areal size of VGluT3 dendritic arbors is inversely proportional to VGluT3 amacrine cell density, a result that would suggest they constrain one another's areal growth in a density-dependent manner.

Single VGluT3 amacrine cells were targeted for intracellular injection with Lucifer yellow by virtue of tdTomato expression in VGluT3-cre reporter mice (Fig. 5a,b). Retinas were subsequently immunolabeled to confirm the VGluT3-positive status of each injected cell, while also permitting the identification of all the other homotypic cells (Fig. 5c), allowing us to compare the size 
of the dendritic field to the density of cells within the sampled field. From that latter population of VGluT3-amacrine cells, the Voronoi tessellation of the field was also constructed (Fig. $5 c$ ), and the area of the dendritic field of the injected cell was then compared directly to its VD (Fig. $5 d$ ). As the VD area of a cell is the inverse of local density at the cell itself, we could therefore examine this local relationship directly (e.g., Weltzien et al., 2014).

Sixty-six well-filled cells that subsequently showed effective immunolabeling of the retina were examined (six more of which are shown in Fig. 5e), sampled across multiple eccentricities from 0.4 to $1.6 \mathrm{~mm}$ from the optic nerve head. These cells had an average dendritic field area of $4723 \pm 120 \mu \mathrm{m}^{2}$. Despite the large variation in dendritic field area across this sample of cells (from 2410 to $6739 \mu \mathrm{m}^{2}$ ), there was no systematic variation with retinal eccentricity (Fig. 6a). The number of immunolabeled cells within the sampled field containing each injected cell was used to compute the density of that field (averaging $1114 \pm 18 \mathrm{cells} / \mathrm{mm}^{2}$ ), which showed a slight decline as a function of eccentricity (Fig. $6 b$ ). Critically, dendritic field area showed no correlation with the variation in cellular density within those individual fields (Fig. 6c).

\section{Proximity to homotypic neighbors does not modulate VGluT3 dendritic areal growth in the WT retina}

There is, however, some degree of immediate variability in the local intercellular spacing for each cell within the mosaic (e.g., Fig. $5 c$ ), and so we examined this relationship more closely, by comparing the dendritic fields of individual cells to their VDs. Even at this local level, however, dendritic field area was independent of VD area (Fig. $6 d$ ). We confirmed the expected negative correlation between VD area and the density of cells within each sampled field (Fig. 6e), but the correlation was not particularly strong, again likely because of the variability in the intercellular spacing present in the VGluT3 amacrine cell mosaic mentioned above (i.e., neighboring VDs in a patch of retina can still vary in size; e.g., Figs. $2 a, 5 \mathrm{c}$ ).

VGluT3 amacrine cells exhibit variable dendritic arbors, some being relatively symmetric around the soma (e.g., Fig. $5 d$ ), whereas others were notably skewed to one side (e.g., Fig. 5e). We therefore examined, for each filled cell, whether the orientation of the dendritic arbor might be related to the distribution of other neighboring cells. Specifically, we counted the number of VGluT3 amacrine cells that fell within the area of the dendritic arbor of each cell, and then systematically rotated the dendritic arbor $90^{\circ}, 180^{\circ}$, and $270^{\circ}$, repeating this count for each of the three rotations (Fig. 6f). VGluT3 amacrine cell dendritic fields were found to enclose, on average, the somata of five other cells, although exhibiting considerable variability in this number, from 1 to 10 cells. These statistics were no different when rotating the dendritic arbors (Fig. 6g), showing comparable cellular overlap. This measure, being an alternative means of estimating dendritic coverage, did however show a strong positive correlation with dendritic field area (Fig. 6h). Rather than modulating dendritic areal growth in relation to any local variation in homotypic presence, other factors must be controlling the total areal size of the dendritic field in the WT retina, and the greater that growth, the more likely the dendritic field overlaid additional homotypic cells.

\section{Dendritic field areas are slightly smaller in the $B a x$-KO retina}

We next examined the organization of dendritic arbors in the Bax-KO retina, where cell density undergoes a threefold increase,
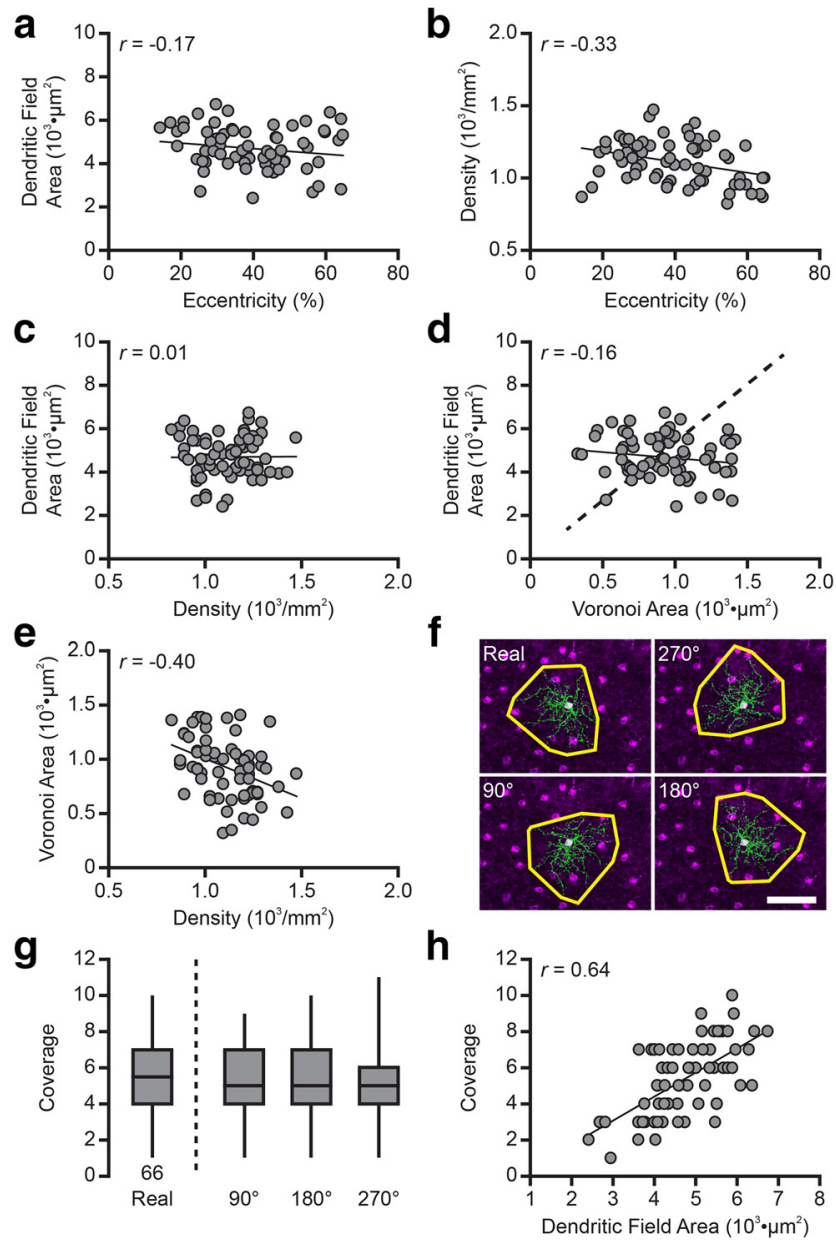

Figure 6. VGluT3 amacrine cell dendritic morphology is unrelated to homotypic cell density or positioning. $\boldsymbol{a}-\boldsymbol{c}$, Scatterplots showing the relationships between VGluT3 cell dendritic field area and retinal eccentricity $(\boldsymbol{a})$, VGluT3 cell density and retinal eccentricity $(\boldsymbol{b})$, and dendritic field area and VGluT3 cell density (c), all from WT retinas. $\boldsymbol{d}$, Scatterplot showing the relationship between dendritic field area and the VD area of each individual cell, being the inverse of cell density at the labeled cell itself. Dashed line indicates the expected size of a dendritic arbor for a cell with a given VD size, assuming these cells maintain a constant coverage of 5.26. $\boldsymbol{e}$, VD area is negatively correlated to field density, but the relationship is loose, consistent with the fact that the mosaic of VGluT3 amacrine cells exhibits occasional irregularities (i.e., neighboring VDs can vary in size because of some local variation in intercellular spacing). $\boldsymbol{f}$, Dendritic fields were systematically rotated relative to the field of cells (shown here for the cell from Fig. $4 a-d$ ), to assess their relationship to the positioning of neighboring cells. $\boldsymbol{g}$, The number of somata covered by each dendritic field (coverage) varied widely within the sample of labeled cells and was unrelated to the orientation of the dendritic field. $\boldsymbol{h}$, Scatterplot showing the relationship between somal coverage and dendritic area. There is a strong positive correlation, indicating that VGluT3 amacrine cells do not modulate their growth to ensure uniformity in their somal overlap. Box plots represent medians and quartiles. $n=$ the number of labeled cells analyzed in $\boldsymbol{g}$. Scale bar, $50 \mu \mathrm{m}$.

well in excess of that observed in the WT retina or in any of the RI strains. We injected 42 Bax-WT cells and 36 Bax-KO cells derived from the two retinas of each of 3 mice, and performed the same morphometric analysis as before, while blinding the experimenter to the genotype of each labeled cell before being analyzed. We observed a significant reduction in the overall areal size of dendritic arbors in the Bax-KO retina, by $29 \%$, despite the large variability within each group (Fig. $7 a, c, d)$. This reduction could not be explained by location on the retina, as cells from both groups were sampled across a comparable breadth of eccentricities from the optic nerve head (not shown). And while the average areal size of dendritic fields was smaller in the Bax-KO 

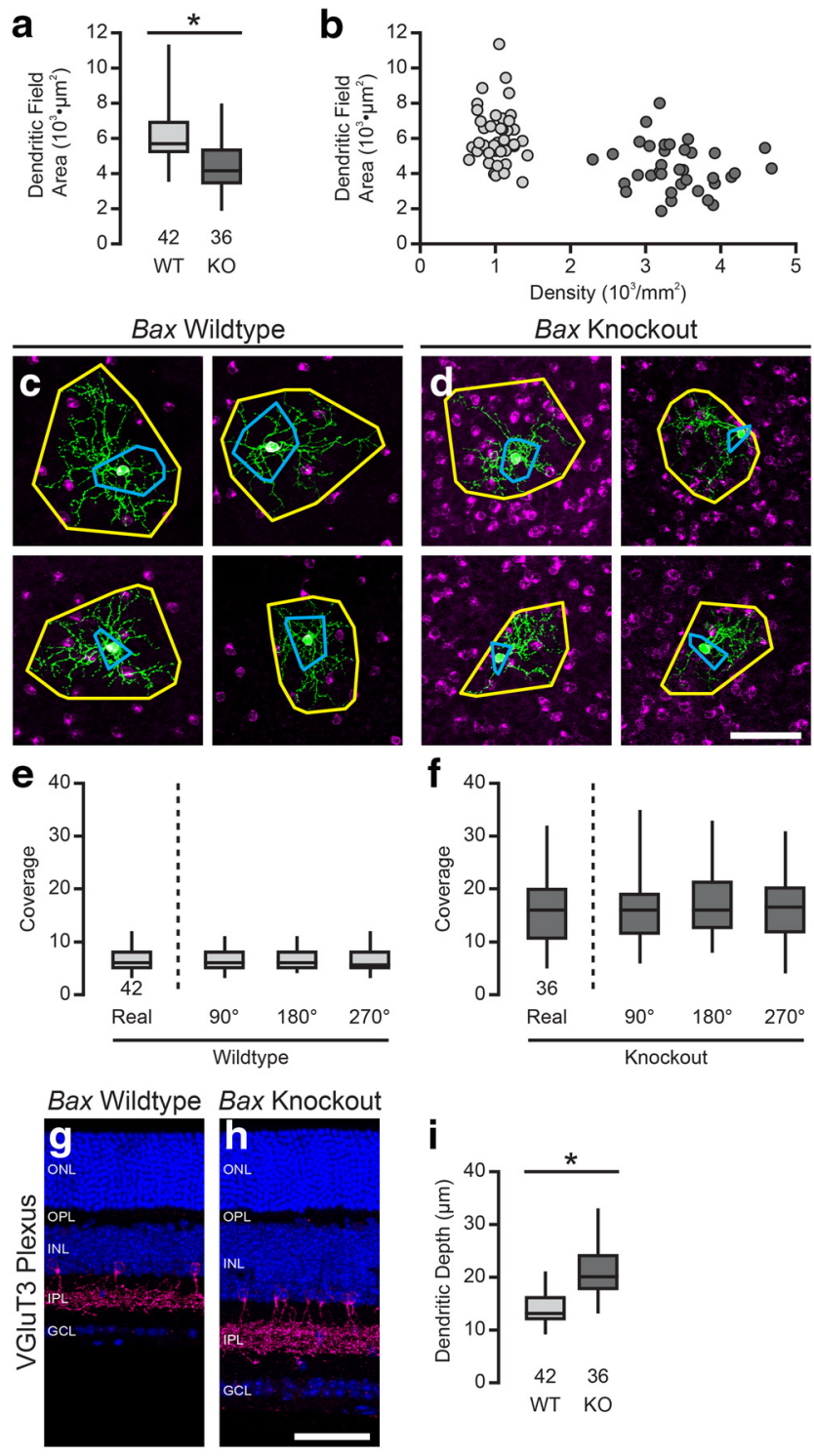

i
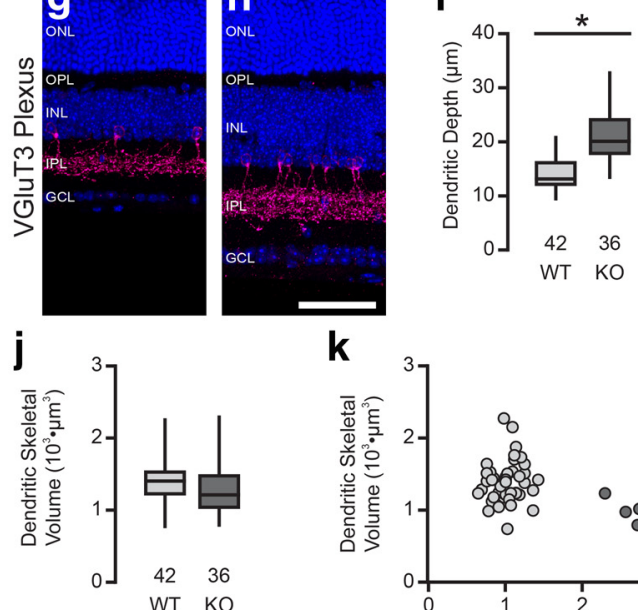

\begin{abstract}
k
\end{abstract}

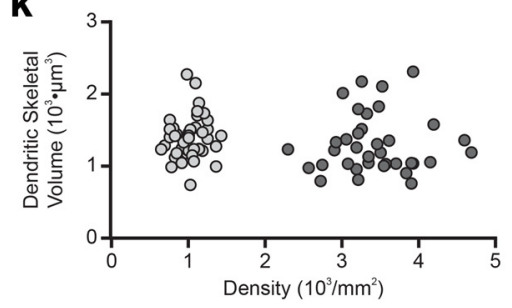

Figure 7. VGluT3 amacrine cells in the Bax-KO retina exhibit a nearly threefold increase in their dendritic coverage. $\boldsymbol{a}$, Single Lucifer yellow-filled cells were sampled from Bax-WT and Bax-KO retinas revealing significantly smaller dendritic field areas for $\mathrm{KO}$ cells $(p<0.001)$. $\boldsymbol{b}$, Scatterplot showing the relationship between dendritic field area and local density of VGluT3 amacrine cells. The decrease in average dendritic field size in the $\mathrm{KO}$ is not proportional to the increase in cell density. $\boldsymbol{c}, \boldsymbol{d}$, Examples of labeled dendritic arbors from both WT (c) and $\mathrm{KO}$ (d) retinas. Also shown is the positioning of all other VGluT3-positive somata within each field. $\boldsymbol{e}, \boldsymbol{f}$, The dendritic coverage of individual cells in the WT $(\boldsymbol{e})$ and KO ( $\boldsymbol{f})$ retinas (defined, as in Fig. $5 f, g$, as the number of somata falling within the convex polygon enclosing the dendritic arbor of each (ell). The reduction in dendritic field area is not sufficient to conserve the average dendritic coverage observed in WT retinas, being $\sim 3$ times higher in the Bax-KO retina $(\boldsymbol{f})$. Rotating each dendritic arbor does not alter the coverage in the KO retina, as described in Figure $5 g$ for cells in the WT retina (and shown here for this sample, in $\boldsymbol{e}$ ). $\boldsymbol{g}, \boldsymbol{h}$, 10 - $\mu$ m-thick cross-sections of retina immunolabeled for VGluT3 (magenta) and stained with Hoechst (blue) to show the distribution of VGluT3-positive processes within the IPL of BaxWT $(\boldsymbol{g})$ and Bax-KO $(\boldsymbol{h})$ retinas. $\boldsymbol{i}$, As expected, individual cells in the Bax-KO extended through a greater depth within the expanded IPL $(p<0.001)$. $\boldsymbol{j}$, The total dendritic skeletal retinas, the reduction was not proportional to the increase in cell density (Fig. $7 b$ ), and thus did not serve to maintain a constant dendritic overlap of neighboring somata: the dendritic coverage increased threefold on average, yet again showing no dependency on the orientation of the dendritic arbor, as in the WT retina (Fig. $7 e, f$ ).

These results indicate that, in the presence of substantially greater numbers of VGluT3 cells in the Bax-KO retina, dendritic arbors do not extend as widely across the tangential extent of the IPL as in the WT retina, but such reduction in their extent does not establish a comparable average dendritic coverage. The plexus of VGluT3-immunopositive processes, arising from those single VGluT3 amacrine cell dendritic arbors, occupies a broader extent of the depth of the retina in Bax-KO mice, as the entire IPL is itself thicker (Fig. $7 g, h$ ). We considered, therefore, whether this increased depth of stratification compensated for the reduction in areal growth to conserve the total dendritic arbor size of these cells. To examine this, the overall size of each arbor (the dendritic skeletal volume) was determined by summing the volume of dendritic processes contained in every optical section through the entire IPL. We found, as expected, that the dendritic arbors in the Bax-KO extend through a greater depth within the IPL (Fig. 7i), and these taller dendritic arbors had comparable dendritic skeletal volumes to those in WT retinas (Fig. 7j); like the dendritic field areal measurements, the skeletal volume of the dendritic arbor was also unrelated to the density of homotypic cells (Fig. $7 k$ ). Together, these results indicate that the overall size of the dendritic arbor is not regulated by homotypic neighbors.

It is noteworthy, however, that VGluT3 cells exhibit considerable variability in the size of their dendritic arbors, both areally in their projection fields as well as in three dimensions, defined by their skeletal volumes (Fig. $7 a, b$ and Fig. $7 j, k$, respectively). Clearly, these cells do not seek to grow in such a manner to achieve arbors of a particular size; rather, their final size is likely influenced by stochastic (albeit undefined) factors. Still, by establishing arbors of comparable size (on average) over a greater radial extent, cells in the Bax-KO should contribute less dendrites to any particular plane of the IPL. Might homotypic neighbors, therefore, be modulating the frequency and patterning of their dendritic branching at each depth to achieve a uniformity in coverage?

\section{Branching frequency of VGluT3 dendritic arbors declines in the Bax-KO retina}

Because of the increased depth of the VGluT3 plexus in the BaxKO retina, the density of immunopositive processes is greater in $z$ axis maximum projection reconstructions (Fig. 8a,c). If, however, single optical sections are directly compared, the VGluT3 plexus exhibits a comparable density (Fig. $8 e, g$ ). We consequently examined the contribution of each labeled dendritic arbor to the immunopositive plexus (Fig. 8b,d) in these same optical sections, finding it to be reduced in the Bax-KO retinas (Fig. $8 f, h)$. We quantified these features within a single optical section for each plexus and for the associated dendritic field within it, for 30 Bax-WT cells and 34 Bax-KO cells that had exceptional

volume was consequently found to be comparable between Bax-WT and Bax-KO retinas. $\boldsymbol{k}_{\boldsymbol{f}}$ Scatterplot showing the relationship between dendritic skeletal volume and local density of VGluT3 amacrine cells, indicating no relationship. ONL, Outer nuclear layer; OPL, outer plexiform layer; $\mathrm{GCL}$, ganglion cell layer. Box plots represent medians and quartiles. $n=$ the number of labeled cells analyzed in $\boldsymbol{a}, \boldsymbol{e}, \boldsymbol{f}, \boldsymbol{i}, \boldsymbol{j}$. Scale bar, $50 \mu \mathrm{m}$. $* p<0.05$. 

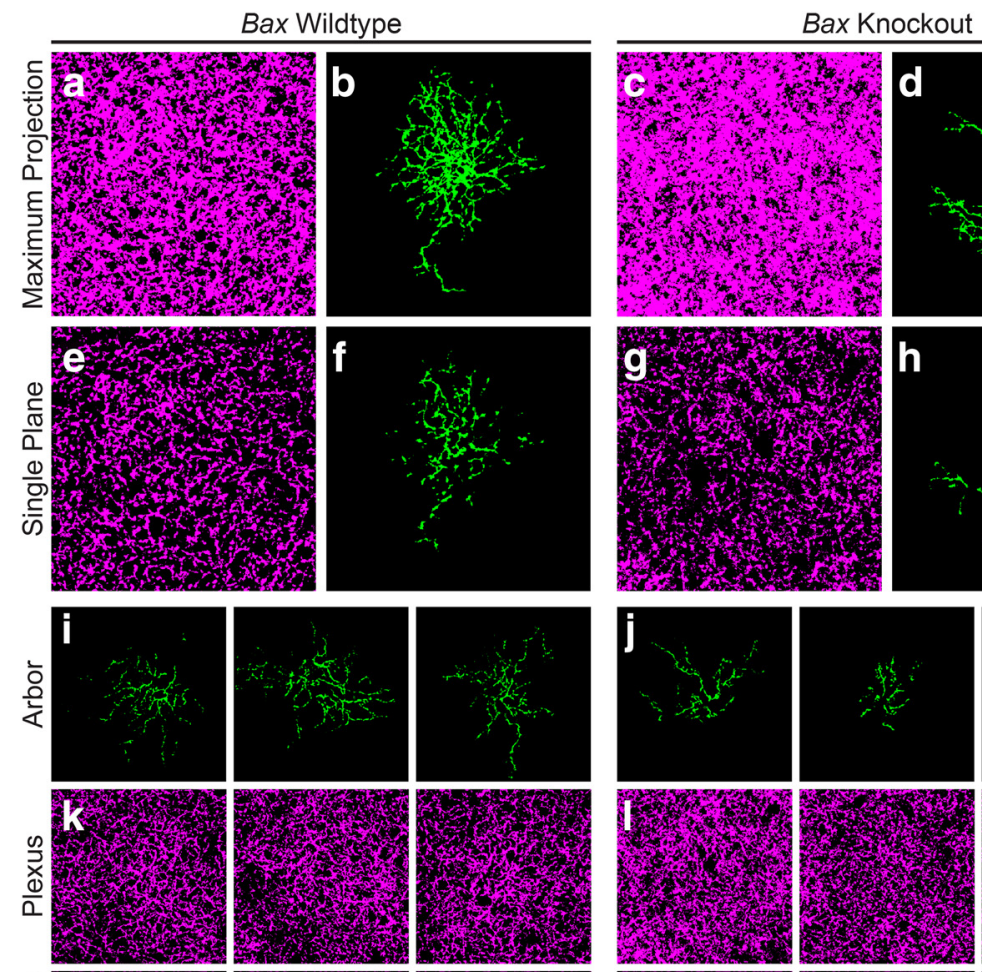
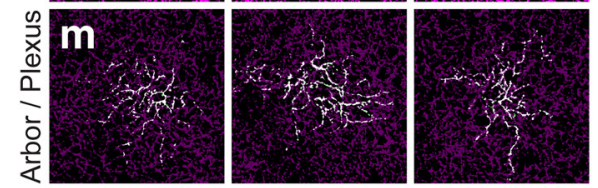

O
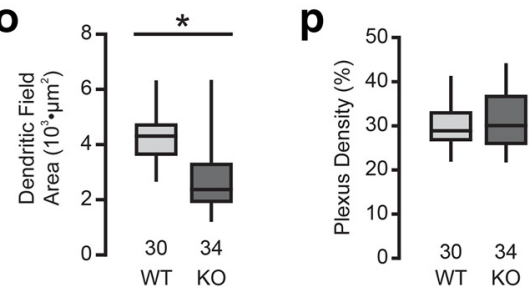
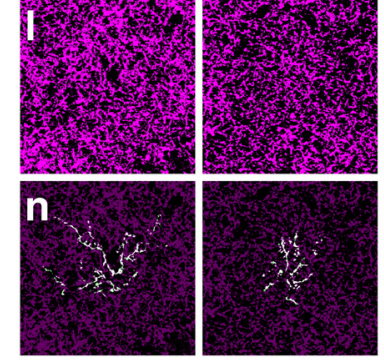

q

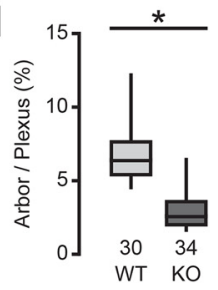

Figure 8. Single VGluT3 dendritic arbors modulate their branching to accommodate the increase in dendritic overlap. $\boldsymbol{a}, \boldsymbol{c}, \boldsymbol{e}$ $\boldsymbol{g}, \boldsymbol{z}$ axis projection through the full depth of the immunolabeled VGluT3 plexus in a wholemounted retina $(\boldsymbol{a}, \boldsymbol{c})$, contrasted with a single optical section $(\boldsymbol{e}, \boldsymbol{g})$ of that same field. $\boldsymbol{b}, \boldsymbol{d}, \boldsymbol{f}, \boldsymbol{h}, \boldsymbol{z}$ axis projection through the full depth of a single dendritic arbor from the same fields shown to the left $(\boldsymbol{b}, \boldsymbol{d})$, or through the same single optical section $(\boldsymbol{f}, \boldsymbol{h})$. The entire plexus is denser in the Bax-KO retina $(\boldsymbol{a}, \boldsymbol{c})$ because of its thicker distribution (made clear in Fig. $7 g, h)$, but that at the level of single optical sections, the plexus density is comparable $(\boldsymbol{e}, \boldsymbol{g}) . \boldsymbol{i}-\boldsymbol{n}$, Three additional examples of single optical sections showing the dendritic arbor of individual cells $(\boldsymbol{i}, \boldsymbol{j})$, the distribution of the immunopositive plexus $(\boldsymbol{k}, \boldsymbol{l})$, and superimposition of the arbor on a muted plexus for better resolution of the former $(\boldsymbol{m}, \boldsymbol{n})$ from Bax-WT $(\boldsymbol{i}, \boldsymbol{k}, \boldsymbol{m})$ and Bax-KO retinas $(\boldsymbol{j}, \mathbf{l}, \boldsymbol{n}) . \mathbf{0}$, The dendritic field area of the arbors in these single planes exhibited a $39 \%$ reduction $(0)(p<0.001)$, which was even more reduced than when calculated from $z$ axis projections (see Figure 7a). $\boldsymbol{p}, \boldsymbol{q}$, The plexus density in single optical sections was comparable in the two conditions $(\boldsymbol{p})(p=0.24)$, whereas the proportion of that plexus contributed by the single-labeled cell was significantly lower for cells in the Bax-KO retina (q) $(p<0.001)$. All micrographs are binary (thresholded) images. Box plots represent medians and quartiles. $n=$ the number of cells analyzed in $\mathbf{0}, \boldsymbol{p}$. Scale bars, $50 \mu \mathrm{m}$. $* p<0.05$.

labeling of both the dendritic tree as well as the immunopositive plexus. As expected, the dendritic field area in these single optical sections was smaller in the Bax-KO, by $39 \%$ (Fig. 8i,j,o), exhibiting an even greater reduction than when viewing the entire arbor in $z$ axis projections (Fig. 7a). Across each sampled optical section, the average density of the VGluT3-immunopositive plexus was comparable in Bax-KO and Bax-WT retinas (Fig. $8 k, l, p$ ), yet the proportion of that labeled plexus occupied by the individual dendritic arbor was significantly reduced, by $\sim 50 \%$ in the BaxKO retina (Fig. $8 m, n, q$ ).
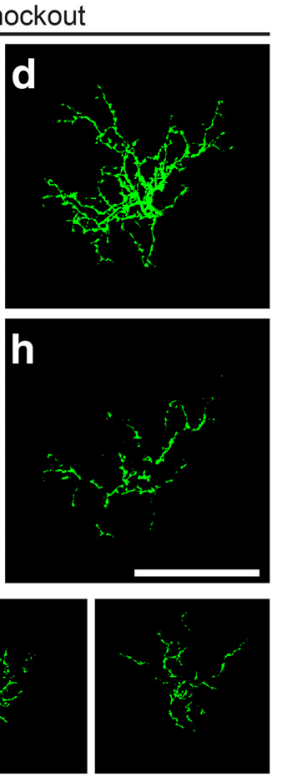

These results indicate that the extent of dendritic branching for single VGluT3-immunopositive cells in the Bax-KO retina does indeed decline, at any depth within the IPL, in the presence of the approximately threefold increase in the number of areally overlapping dendritic arbors. Such a decline would therefore suggest that the processes of neighboring cells must be coordinated to yield a comparable volumetric density. If true, any local variation in plexus density should be unrelated to the degree of irregularity in somal spacing.

\section{Local variation in VGluT3 plexus density is independent of underlying cellular positioning} The foregoing results, showing a reduction in the contribution of single VGluT3 dendritic arbors at a particular depth within the Bax-KO retina, suggest that neighboring cells modulate process growth to achieve a uniformity in the density of the entire plexus. The density of the VGluT3immunopositive plexus appears uniform at any depth, yet there is clearly occasional local variation in the density of processes across a field (Fig. $8 e, g)$. Given the lack of correlation between dendritic arbor area and VD area (Fig. $6 d$ ), as well as the substantial variation in dendritic coverage (Fig. $6 h$ ), might that local variation within the immunopositive plexus simply reflect a random positioning of multiple overlapping dendritic arbors?

If dendritic growth within the plexus is coordinated between dendrites from homotypic neighbors, we would expect plexus density to be invariant across a given field, regardless of the patterning present among the VGluT3 amacrine cells themselves (Fig. 9a,b). By contrast, if cells position their dendrites at random, then such local variability in plexus density across a field would be expected to increase as the arrangement of somata becomes less regular. To assess this feature of the VGluT3 plexus, we divided each field within these single optical sections into 16 subfields, then determined the thresholded plexus density of each subfield to examine the extent to which this density varies across the entire field (Fig. $9 c, d$ ). We assessed this by determining the coefficient of variation $(\mathrm{CoV})$ for each field (being the SD divided by the mean): the lower the $\mathrm{CoV}$, the more evenly distributed the plexus is across the field. The average $\mathrm{CoV}$ was $0.17 \pm 0.01$ for KO fields, comparable to that for WT fields, being $0.18 \pm 0.01$ (Fig. 9e), despite the differences in the organization of their somata (Fig. 9a,b). Furthermore, the $\mathrm{CoV}$ 

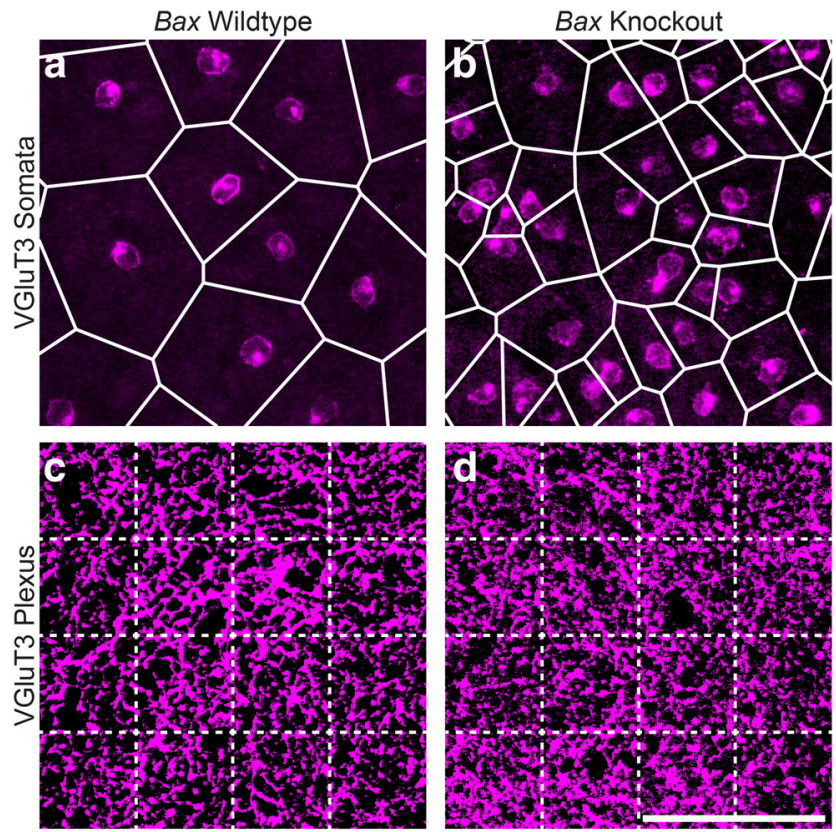
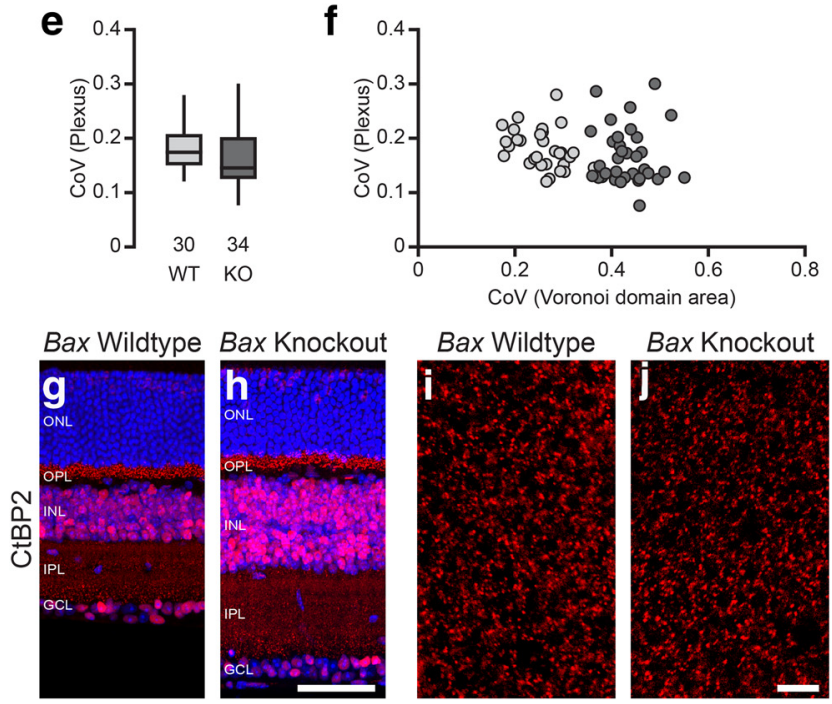

Figure 9. The uniformity of the VGluT3-immunopositive plexus across the IPL is not affected by a loss of somal patterning. $\boldsymbol{a}, \boldsymbol{b}$, VGluT3 somata in Bax-WT (a) and Bax-KO (b) retinas, displaying the characteristic differences in their mosaic regularity evident in the Voronoi tessellations. If dendrites grow without regard to their homotypic neighbors, then the variability in the density of the VGluT3 plexus would be expected to be higher in fields with less regular somal arrays. $\boldsymbol{c}, \boldsymbol{d}$, Single optical sections of the VGluT3 plexus from the fields shown in $\boldsymbol{a}$ and $\boldsymbol{b}$, respectively, divided into 16 subfields, from which plexus density was determined for each of the sampled fields, and the CoV across these 16 subfields was calculated. $\boldsymbol{e}$, There was no significant difference in the average $\mathrm{CoV}$ between the two genotypes $(p=0.25)$. $\boldsymbol{f}$, The CoV for the VD areas associated with the somata in the same fields was also computed, showing no trend of a positive correlation to the CoV for plexus density. $\boldsymbol{g}, \boldsymbol{h}, 10-\mu \mathrm{m}$-thick cross-sections of retina immunolabeled for CtBP2 (red) and stained with Hoechst (blue) to show the distribution of presynaptic CtBP2-postive ribbon synapses within the IPL of Bax-WT $(\boldsymbol{g})$ and Bax-KO $(\boldsymbol{h})$ retinas. $\boldsymbol{i}, \boldsymbol{j}$, Distribution of CtBP2 puncta in a single optical plane through the middle of the IPL from Bax-WT $(\boldsymbol{g})$ and Bax-KO $(\boldsymbol{h})$ retinal wholemounts. ONL, Outer nuclear layer; OPL, outer plexiform layer; $\mathrm{GCL}$, ganglion cell layer. $\boldsymbol{c}, \boldsymbol{d}$, Micrographs are binary (thresholded) images. Box plots represent medians and quartiles. $n$ $=$ the number of fields analyzed in $\boldsymbol{e}$. Scale bars: $\boldsymbol{a}-\boldsymbol{d}, \boldsymbol{g}, \boldsymbol{h}, 50 \mu \mathrm{m} ; \boldsymbol{i}, \boldsymbol{j}, 10 \mu \mathrm{m}$.

of the plexus density was not correlated to the variation of their somal mosaics as assessed by determining the $\mathrm{CoV}$ of their VD areas (Fig. 9f). VGluT3 amacrine cells, therefore, do not randomly position their dendrites in the IPL; rather, they must modulate their branching at the local level to approximate a uniformity in process density across the population.

Exactly where these cells position their dendritic arbors within the IPL is doubtless influenced by their synaptic partners as they too expand across a greater depth in the Bax-KO retina. Given our observation that bipolar cells regulate the number and patterning of this cell population, it is possible that they play a role in instructing or permitting the growth of VGluT3 amacrine cell dendrites. Yet the relative constancy in the density of the VGluT3-immunopositive plexus, where the density and variability of ribbon synapses appear to be unchanged in the Bax-KO retina (Fig. $9 g-j$ ), is consistent with the view that the positioning of the dendritic branches of individual VGluT3 amacrine cells is modulated by the presence of those of their homotypic neighbors to yield that constancy across the retina. Whether the mechanism driving this uniformity is direct, acting between neighboring cells, or indirect, because of competition for afferents or targets, remains unknown; it is clear, however, that the unique branching patterns of single cells must relate to those of their homotypic neighbors to ensure a uniformity in the VGluT3-immunopositive plexus.

\section{Discussion}

The present study demonstrates four main findings: First, Baxmediated cell death in the B6/J retina is responsible for establishing the mature number of VGluT3 amacrine cells in their characteristically regular somal array. Second, genetic variants discriminating the B6/J and A/J strains of mice affect both the density of VGluT3 cells and their mosaic regularity, but do so independent of one another, indicating other modulators of both traits contribute, at least when Bax is intact. Third, the Bax dependency of VGluT3 amacrine cell number is shown to be mediated by afferent support provided by the bipolar cell population. And fourth, VGluT3 cells have dendritic areal sizes that bear no spatial relationship to the local variation in VGluT3 cell density or positioning, yet modulate their overlapping dendritic branches in relation to homotypic density. We consider below the conclusions that can be drawn relating these four sets of studies for understanding the determinants of these demographic properties of VGluT3 amacrine cells.

\section{Programmed cell death reduces cell density while increasing} mosaic regularity

Our first study disrupted the function of a gene modulating a known biological process, naturally occurring cell death. BaxKO mice were found to have populations of VGluT3 cells that showed a greater than threefold increase in density relative to littermate controls. Curiously, rather than exhibiting a spatial distribution in which these cells minimize proximity to neighboring homotypic cells, as they approximate in the WT retina, VGluT3 cells in the Bax-KO retina were nearly as irregular in their distribution as observed in random simulations matched in density. As elimination of Bax is thought to preserve a proportion of postmitotic cells that would otherwise have died during development, these results from the Bax-KO retina would suggest that the initial production of VGluT3 cells yields a population that is randomly distributed, and cell death preferentially serves to eliminate close neighbors. Indeed, our simulations of proximitybased cell death commensurate with this magnitude of cell loss yield distributions roughly comparable to those of the Bax-WT retina. 


\section{Cell density and mosaic patterning can be modulated independent of each other}

Our second study took advantage of the large variation in the size of the VGluT3 population across a panel of RI strains (Keeley et al., 2014a). These strains are all derived from two inbred laboratory strains $(\mathrm{B} 6 / \mathrm{J}$ and $\mathrm{A} / \mathrm{J})$, yet the genome of each of the $26 \mathrm{RI}$ strains is uniquely comprised of distinct B6/J versus A/J haplotype blocks along each chromosome. Such RI strain analysis provides a potentially powerful way to locate the genomic sources of such variation in traits through QTL mapping, as well as asking whether pairs of traits are correlated across the strains and whether they share controlling genomic loci. Our expectation, in light of the first study, was that we might find a negative correlation between cell density and regularity across the strains, suggesting that the robustness by which cell death reduces cellular density might also yield a corresponding increase in the uniformity of intercellular spacing, thereby generating greater regularity. In fact, we detected only a hint of a negative correlation between cell density and regularity in the VGluT3 cells across these strains, nor did we see any suggestion of shared genomic control of these two traits.

From the above Bax-KO analysis, we would expect that all of these RI strains overproduce the population of VGluT3 cells during normal development, and that a wave of programmed cell death reduces their numbers. We would also conclude that the initial production of these cells yields an early distribution that is comparably random yet becomes more regular through this cell death. As the variation in neither trait maps to chromosome 7, where Bax is located, we would further conclude that little of the variability of either of these two traits for this cell type is associated with sequence variants in Bax (Whitney et al., 2009). Rather, we must conclude that other variant genes independently modulate both cell density and mosaic regularity, but whether they act through the same biological process or two independent processes is unclear. For instance, tangential migration may also play a role in modulating the mosaic regularity for this cell type (Reese et al., 1999).

\section{Bax-dependent VGluT3 cell death is mediated via bipolar afferents}

We subsequently asked whether the Bax-mediated effect on VGluT3 cell density and patterning was because of a cell-intrinsic effect of Bax. Many, but not all, retinal cell types have their numbers increased in the Bax-KO retina; few studies, however, have analyzed which of those cell types are modulated via a cell-intrinsic action of Bax versus an action within either their afferents or their targets (Keeley et al., 2014b). Here, we have examined the effect of deleting Bax selectively, in either the VGluT3 amacrine cells themselves, or within their target retinal ganglion cells or their afferent bipolar cells, using three different Cre-expressing lines of mice.

In the VGluT3-specific CKO retina, this cell type showed no alteration in cell number whatsoever, which may suggest that Bax deletion from VGluT3 amacrine cells does not enhance their survival. We acknowledge, however, the possibility that Cre is not present until too late, after the wave of Bax-dependent cell death has already reduced the number of VGluT3 amacrine cells, as crossing this VGluT3-cre line with Cre-reporter mice showed tdTomato labeling in these cells only after P8, consistent with the fact that immunostaining for VGluT3 reveals positive cells only after P7. Absent a Cre-expressing line that turns on selectively in
VGluT3 cells shortly after their genesis, we cannot resolve this uncertainty. By contrast, the bipolar cell-specific CKO retina showed a large effect on VGluT3 cell numbers, if not quite as large as the effect in the Bax-KO retina, while the ganglion cellspecific CKO retina showed no effect. The Bax-mediated effect on VGluT3 cell numbers in the Bax-KO retina, therefore, reflects an afferent, but not target, dependency. All bipolar cell types should express Vsx2 (Nickerson et al., 2011; Shekhar et al., 2016), but not all bipolar cell types may provide afferent support critical for survival within the VGluT3 cell population. While we cannot yet account for the discrepancy in total numbers of VGluT3 cells between this $\mathrm{CKO}$ and the Bax-KO, the effect is nonetheless large and unexpected. In these retinas, restricting the loss of Bax to bipolar cells, not only the number but also the patterning of VGluT3 cells was altered, the latter achieving a regularity ratio associated with a random distribution (Fig. 4p). As multiple bipolar cell types are afferent to VGluT3 amacrine cells, and each bipolar cell type is thought to be assembled as a tiling mosaic (Wässle et al., 2009) of randomly distributed somata (Keeley et al., 2020), these results suggest a trophic effect by the bipolar afferent terminals on VGluT3 amacrine cell survival during development that normally yields closely spaced VGluT3 cells at a greater disadvantage for surviving (i.e., the spatial positioning of bipolar somata should play no role on the spacing of the VGluT3 cells).

\section{Dendritic arbor size is not constrained by homotypic neighbors in the WT retina}

That this population of cells shows a modulation of both density as well as patterning might lead one to question the ramifications for dendritic coverage. Where neither the density of cells, nor their mosaic spacing, is tightly controlled across strains in the mature mouse retina, do these cells modulate their dendritic outgrowth in a compensatory manner, to establish a cell-type specific degree of dendritic coverage, as has been suggested for other cell types, for instance, the horizontal cells (Reese et al., 2005)?

We first assessed the variation in dendritic field area in the WT retina as a function of regional cell density. No correlation was observed, despite large variation in dendritic field area between individual cells. Indeed, when we examined the correlation between dendritic field area and the area of the VD for the very same cells, no relationship was detected. Given that the mosaic of VGluT3 cells exhibits some degree of local variability, with asymmetric distributions of homotypic neighbors, we asked whether dendritic arbors showed a tendency to skew their orientation accordingly, yet we observed no such effect. From these results, we conclude that VGluT3 cells establish the areas of their dendritic arbors independent of homotypic constraints. Neighboring VGluT3 cells neither prevent overlap between one another (i.e., they do not tile), nor do they modulate their areal growth in either a density- or proximity-dependent manner in the WT retina.

VGluT3 amacrine cells spread their dendrites into the territories of neighboring cells. They have been estimated to have a dendritic coverage factor of 6.88 (Kim et al., 2015), although our own estimates, based on multiplying average dendritic field area by the average density of cells in those same sampled fields, yield a slightly lower dendritic coverage factor of 5.26, which comes close to the average number of cells falling within each dendritic field (5.38). Of course, such a summary statistic can be 
misleading if it is taken to imply a conservation of this degree of coverage, for in the present case, we have seen that the size of individual dendritic fields bears no relationship to $\mathrm{VD}$ area in the WT retina. Indeed, the sizes of VGluT3 dendritic arbors clearly deviate from the field area required to maintain a coverage factor of 5.26 for any given VD area (Fig. $6 d$, dashed line), as to be expected from the highly variable number of somata falling within these dendritic fields, ranging from 1 to 10 (Fig. 6h).

\section{Dendritic branching is coordinated between homotypic neighbors}

When homotypic density is substantially increased, in the Bax$\mathrm{KO}$, a modest reduction in dendritic area is observed (Fig. 7a), but it does not approximate the scale expected to maintain a comparable degree of dendritic coverage to that observed in the WT retina. The contribution of individual dendrites to any particular plane of the overall plexus, by contrast, declines in the presence of such a large increase in the density of homotypic neighbors (Fig. 8q), suggesting that overlapping dendritic arbors constrain branch frequency. Our analysis of the local variability in VGluT3 plexus density in both Bax-WT and Bax-KO retinas (Fig. 9) supports the hypothesis that dendritic branching must be coordinated between overlapping dendritic arbors, but a direct role for homotypic interactions in mediating this effect could not be assessed in the VGluT3-cre Bax-CKO retina. Regardless, these results suggest that VGluT3 amacrine cells operate according to the density conservation principle (Bae et al., 2018), achieving a coordination of their local branching to approximate uniform process density. The fact that dendritic processing by VGluT3 cells is locally isolated (Chen et al., 2017; Hsiang et al., 2017) would appear consistent with such a growth strategy, where the areal extent of a dendritic arbor, and the degree to which it overlies neighboring arbors in the plane of the retina, may have no functional significance.

\section{References}

Bae JA, Mu S, Kim JS, Turner NL, Tartavull I, Kemnitz N, Jordan CS, Norton AD, Silversmith WM, Prentki R, Sorek M, David C, Jones DL, Bland D, Sterling AL, Park J, Briggman KL, Seung HS, Eyewirers (2018) Digital museum of retinal ganglion cells with dense anatomy and physiology. Cell 173:1293-1306.

Chen MG, Lee S, Zhou ZJ (2017) Local synaptic integration enables ON-OFF asymmetric and layer-specific visual information processing in vGluT3 amacrine cell dendrites. Proc Natl Acad Sci USA 114:11518-11523.

Diamond JS (2017) Inhibitory interneurons in the retina: types, circuitry, and function. Annu Rev Vis Sci 3:1-24.

Farajian R, Raven MA, Cusato K, Reese BE (2004) Cellular positioning and dendritic field size of cholinergic amacrine cells are impervious to early ablation of neighboring cells in the mouse retina. Vis Neurosci 21:13-22.

Grimes WN, Seal RP, Oesch N, Edwards RH, Diamond JS (2011) Genetic targeting and physiological features of VGLUT3 $^{+}$amacrine cells. Vis Neurosci 28:381-392.

Haverkamp S, Wassle H (2004) Characterization of an amacrine cell type of the mammalian retina immunoreactive for vesicular glutamate transporter 3. J Comp Neurol 468:251-263.

Hsiang JC, Johnson KP, Madisen L, Zeng HK, Kerschensteiner D (2017) Local processing in neurites of VGluT3-expressing amacrine cells differentially organizes visual information. eLife 6:e31307.

Huckfeldt RM, Schubert T, Morgan JL, Godinho L, Di Cristo G, Huang ZJ, Wong RO (2009) Transient neurites of retinal horizontal cells exhibit columnar tiling via homotypic interactions. Nat Neurosci 12:35-43.

Jia Y, Lee S, Zhuo Y, Zhou ZJ (2020) A retinal circuit for the suppressed-bycontrast receptive field of a polyaxonal amacrine cell. Proc Natl Acad Sci USA 117:9577-9583.

Johnson J, Sherry DM, Liu XR, Fremeau RT, Seal RP, Edwards RH, Copenhagen DR (2004) Vesicular glutamate transporter 3 expression identifies glutamatergic amacrine cells in the rodent retina. J Comp Neurol 477:386-398.

Keeley PW, Reese BE (2014) The patterning of retinal horizontal cells: normalizing the regularity index enhances the detection of genomic linkage. Front Neuroanat 8:113.

Keeley PW, Reese BE (2018) The somal patterning of the AII amacrine cell Imosaic in the mouse retina is indistinguishable from random simulations matched for density and constrained by soma size. Vis Neurosci 35: e003.

Keeley PW, Whitney IE, Madsen NR, St. John AJ, Borhanian S, Leong SA, Williams RW, Reese BE (2014a) Independent genomic control of neuronal number across retinal cell types. Dev Cell 30:103-109.

Keeley PW, Madsen NR, St. John AJ, Reese BE (2014b) Programmed cell death of retinal cone bipolar cells is independent of afferent or target control. Dev Biol 394:191-196.

Keeley PW, Whitney IE, Reese BE (2017a) Genomic control of retinal cell number: challenges, protocol, and results. In: Methods in molecular biology: systems genetics (Schughart K, Williams RW, eds), pp 365-390. New York: Springer.

Keeley PW, Kim JJ, Lee SC, Haverkamp S, Reese BE (2017b) Random spatial patterning of cone bipolar cell mosaics in the mouse retina. Vis Neurosci 34:e002.

Keeley PW, Eglen SJ, Reese BE (2020) From random to regular: variation in the patterning of retinal mosaics. J Comp Neurol 528:2135-2160.

Kim T, Soto F, Kerschensteiner D (2015) An excitatory amacrine cell detects object motion and provides feature-selective input to ganglion cells in the mouse retina. eLife 4:e08025.

Lee S, Zhang Y, Chen MG, Zhou ZJ (2016) Segregated glycine-glutamate cotransmission from vGluT3 amacrine cells to contrast-suppressed and contrast-enhanced retinal circuits. Neuron 90:27-34.

Lee SC, Cowgill EJ, Al-Nabulsi A, Quinn EJ, Evans SM, Reese BE (2011) Homotypic regulation of neuronal morphology and connectivity in the mouse retina. J Neurosci 31:14126-14133.

Mosinger-Ogilvie J, Deckwerth TL, Kundson CM, Korsmeyer SJ (1998) Suppression of developmental retinal cell death but not photoreceptor degeneration in Bax-deficient mice. Invest Ophthalmol Vis Sci 39:17131720.

Nickerson PE, Ronellenfitch K, McEwan J, Kim H, McInnes RR, Chow RL (2011) A transgenic mouse line expressing cre recombinase in undifferentiated postmitotic mouse retinal bipolar cell precursors. PLoS One 6:e27145.

Péquignot MO, Provost AC, Sallé S, Taupin P, Sainton KM, Marchant D, Martinou JC, Ameisen JC, Jais JP, Abitbol M (2003) Major role of BAX in apoptosis during retinal development and in establishment of a functional postnatal retina. Dev Dyn 228:231-238.

Poché RA, Raven MA, Kwan KM, Furuta Y, Behringer RR, Reese BE (2008) Somal positioning and dendritic growth of horizontal cells are regulated by interactions with homotypic neighbors. Eur J Neurosci 27:16071614.

Puñal VM, Paisley CE, Brecha FS, Lee MA, Perelli RM, Wang J, O'Koren EG, Ackley CR, Saban DR, Reese BE, Kay JN (2019) Large-scale death of retinal astrocytes during normal development is non-apoptotic and implemented by microglia. PLoS Biol 17:e3000492.

Raven MA, Reese BE (2002) Horizontal cell density and mosaic regularity in pigmented and albino mouse retina. J Comp Neurol 454:168-176.

Raven MA, Eglen SJ, Ohab JJ, Reese BE (2003) Determinants of the exclusion zone in dopaminergic amacrine cell mosaics. J Comp Neurol 461:123-136.

Reese BE, Keeley PW (2015) Design principles and developmental mechanisms underlying retinal mosaics. Biol Rev Camb Philos Soc 90:854-876.

Reese BE, Keeley PW (2016) Genomic control of neuronal demographics in the retina. Prog Retin Eye Res 55:246-259.

Reese BE, Necessary BD, Tam PP, Faulkner-Jones B, Tan SS (1999) Clonal expansion and cell dispersion in the developing mouse retina. Eur J Neurosci 11:2965-2978.

Reese BE, Raven MA, Stagg SB (2005) Afferents and homotypic neighbors regulate horizontal cell morphology, connectivity and retinal coverage. J Neurosci 25:2167-2175.

Reese BE, Keeley PW, Lee SC, Whitney IE (2011) Developmental plasticity of dendritic morphology and the establishment of coverage and connectivity in the outer retina. Dev Neurobiol 71:1273-1285. 
Sankaran M, Keeley PW, He L, Iuvone PM, Reese BE (2018) Dopaminergic amacrine cell number, plexus density, and dopamine content in the mouse retina: strain differences and effects of Bax gene disruption. Exp Eye Res 177:208-212.

Shekhar K, Lapan SW, Whitney IE, Tran NM, Macosko EZ, Kowalczyk M, Adiconis X, Levin JZ, Nemesh J, Goldman M, McCarroll SA, Cepko CL, Regev A, Sanes JR (2016) Comprehensive classification of retinal bipolar neurons by single-cell transcriptomics. Cell 166: 1308-1323.

Tien NW, Kim T, Kerschensteiner D (2016) Target-specific glycinergic transmission from VGluT3-expressing amacrine cells shapes suppressive contrast responses in the retina. Cell Rep 15:1369-1375.
Wässle H, Puller C, Müller F, Haverkamp S (2009) Cone contacts, mosaics, and territories of cone bipolar cells in the mouse retina. J Neurosci 29:106-117.

Weltzien F, Dimarco S, Protti DA, Daraio T, Martin PR, Grunert U (2014) Characterization of secretagogin-immunoreactive amacrine cells in marmoset retina. J Comp Neurol 522:435-455.

Whitney IE, Raven MA, Keeley PW, Reese BE (2008) Spatial patterning of cholinergic amacrine cells in the mouse retina. J Comp Neurol 508: 1-12.

Whitney IE, Raven MA, Ciobanu DC, Williams RW, Reese BE (2009) Multiple genes on chromosome 7 regulate dopaminergic amacrine cell number in the mouse retina. Invest Ophthalmol Vis Sci 50:1996-2003. 